\title{
Seismic velocity structure in the Earth's outer core
}

\author{
Wen-che Yu and Lianxing Wen \\ Department of Geosciences, State University of New York at Stony Brook, Stony Brook, New York, USA
}

Fenglin Niu

Department of Earth Science, Rice University, Houston, Texas, USA

Received 4 December 2003; revised 26 October 2004; accepted 22 November 2004; published 24 February 2005.

[1] We constrain seismic velocity structure in the Earth's outer core by analyzing differential travel times, waveforms, and amplitude ratios of several pairs of core phases. We use differential travel times and waveforms of $P K P b c-P K I K P$ at $144^{\circ}-148^{\circ}$, $P K i K P-P K I K P$ at $120^{\circ}-141^{\circ}, P K i K P-P K P B_{\text {diff }}$ at the $P K P$ caustics distance range $\left(141^{\circ}-145^{\circ}\right)$, and differential travel times and amplitude ratios of $P K P a b-P K P b c$ at $146^{\circ}-159^{\circ}$. To avoid the influence of the inner core anisotropy in velocity, we only use the PKiKP-PKIKP and the PKPbc-PKIKP observations whose PKIKP phases sample the inner core along the equatorial paths (paths with their ray angles being larger than $35^{\circ}$ from the Earth's rotation axis). These observations show the following characteristics: (1) both the observed PKPbc-PKIKP and PKiKP-PKIKP differential travel times show a distinct "east-west" hemispheric pattern. PKIKP phases arrive about $0.7 \mathrm{~s}$ earlier for those sampling the "eastern" hemisphere $\left(40^{\circ} \mathrm{E}-180^{\circ} \mathrm{E}\right)$ than those sampling the "western" hemisphere $\left(180^{\circ} \mathrm{W}-40^{\circ} \mathrm{E}\right)$; (2) the observed differential $P K i K P-P K P B_{\text {diff }}$ travel time residuals also exhibit a hemispheric difference. $P K i K P-P K P B_{\text {diff }}$ differential travel times are about $0.9 \mathrm{~s}$ larger for those sampling the western hemisphere than those sampling the eastern hemisphere; and (3) both the observed $P K P a b-P K P b c$ differential travel times and $P K P b c / P K P a b$ amplitude ratios show scatter. Overall, these observations can be best explained by two one-dimensional $P$ velocity models, one for each hemisphere, at the bottom of the outer core. The seismic data sampling the eastern hemisphere can be explained by PREM at the bottom of the outer core, while those sampling the western hemisphere can be explained by a lower velocity gradient at the bottom of the outer core, which has reduced velocities relative to PREM linearly increasing from $0 \%$ at $200 \mathrm{~km}$ above the inner core boundary (ICB) to $-0.35 \%$ at the ICB. Different velocity gradients at the bottom of the outer core indicate that there may exist a compositional difference and/or a large-scale temperature difference there and that inner core formation processes may be different between the two hemispheres. Different inner core formation processes may produce different geometric inclusions of melt in the top of the inner core, and thus may provide an explanation to the hemispheric variation of seismic velocity and attenuation in the top of the inner core. The seismic data also suggest that the variation of seismic velocity in the tangential cylinder of the outer core, if it exists, is less than $0.1 \%$. We use a compressional wave tomographic model to study the travel time delays caused by the seismic heterogeneities in the mantle. The tomographic model is unable to explain the scatter of the travel time data, suggesting the existence of unknown strong small-scale seismic heterogeneities in the mantle.

Citation: Yu, W., L. Wen, and F. Niu (2005), Seismic velocity structure in the Earth's outer core, J. Geophys. Res., 110, B02302, doi:10.1029/2003JB002928.

\section{Introduction}

[2] Seismic velocity models place fundamental constraints on the nature of the Earth's outer core. It has long been argued that the Earth's outer core, due to its low

Copyright 2005 by the American Geophysical Union. 0148-0227/05/2003JB002928\$09.00 viscosity, should be homogeneous from dynamic considerations [Stevenson, 1987]. Seismological models of the outer core are, however, far from conclusive. Several previous studies suggested inhomogeneity may exist in the outermost part of the core [Souriau and Poupinet, 1990, 1991b; Tanaka and Hamaguchi, 1993; Garnero and Helmberger, 1995], within the tangential cylinder or within two caps at the poles of the outermost core [Romanowicz and Breger, 


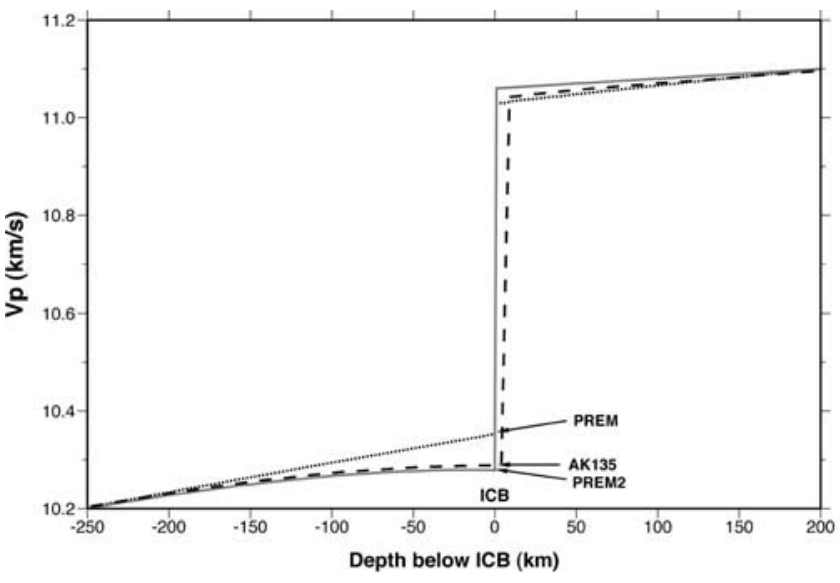

Figure 1. Velocity profiles of three recent $P$ wave models near the Earth's inner core boundary (ICB): PREM [Dziewonski and Anderson, 1981], PREM2 [Song and Helmberger, 1995], and AK135 [Kennett et al., 1995]. Note the different velocity gradients at the bottom $200 \mathrm{~km}$ of the outer core among PREM, PREM2, and AK135.

2000], or at the bottom of the outer core [Souriau and Poupinet, 1991a; Song and Helmberger, 1992]. There is even no consensus on the one-dimensional seismic velocity models for the Earth's outer core. For example, Figure 1 shows velocity profiles of three most recent models of the Earth's core, Preliminary Reference Earth Model (PREM) [Dziewonski and Anderson, 1981], PREM2 [Song and Helmberger, 1995], and AK135 [Kennett et al., 1995]. These models differ significantly at the bottom of the outer core. The discrepancies among these models were resulted from different types of seismic data sets and their sampling coverage used in inferring the models. It becomes important to critically assess the robustness of different model features resulted from different data sets for two reasons: 1) different seismic structures employed in the bottom of the outer core will directly affect the inference of seismic structures in the inner core [e.g., Wen and Niu, 2002]; and 2) the implications to the nature of the outer core among those models are enormously different. For example, while PREM may be reasonably attributed to a homogeneous outer core, PREM2 or AK135 would require existence of compositional change in the bottom of the outer core.

[3] In this study, we explore seismic models in the outer core by studying various $P K P$ observations and attempt to clarify previous propositions to the outer core on the basis of the above seismic observations. We search these data sets globally and use as many different phase pairs as possible, in order to avoid the bias of sampling coverage. It is also important to understand how in practice different types of core phases can be used to constrain the seismic velocity structure in the outer core and how these phases are affected by the seismic heterogeneities elsewhere. We focus mostly on the seismic velocity structure at the bottom of the outer core, where seismic heterogeneities most likely exist and current seismic models differ most. Because our most robust data set in deriving the outer core model is the PKiKP$P K I K P$ waveforms and the PKPbc-PKIKP differential travel times and waveforms, which exhibit an "east-west" hemispheric pattern that is clearly related to the "east-west" hemispheric dichotomy of the seismic structures in the top of the inner core [Niu and Wen, 2001; Wen and Niu, 2002; $Y u$ et al., 2003], we unavoidably focus most of the discussions on the hemispheric dependence of the seismic data. Though, we will also discuss the data dependence on turning latitude, turning longitude, azimuth, ray angle, and the travel path within the tangential cylinder. On the other hand, exploring hemispheric variation in the outer core may also help us to understand the possible cause of the hemispheric dichotomy of seismic velocity and attenuation structures in the top of the inner core.

\section{Seismic Data and Models}

[4] Seismic velocity models in the Earth's outer core will be derived by fitting several $P K P$ observations at the epicentral distance range of $120^{\circ}-159^{\circ}$ : PKiKP-PKIKP differential travel times and waveforms at $120^{\circ}-141^{\circ}$, $P K i K P-P K P B_{\text {diff }}$ differential travel times and waveforms at $141^{\circ}-145^{\circ}, P K P b c-P K I K P$ differential travel times and waveforms at $144^{\circ}-153^{\circ}, P K P a b-P K P b c$ differential travel times at $146^{\circ}-159^{\circ}$, and $P K P b c / P K P a b$ amplitude ratios at $146^{\circ}-159^{\circ}$. To avoid the influence of the inner core anisotropy in velocity, we only use the PKiKP-PKIKP and the $P K P b c-P K I K P$ observations whose $P K I K P$ phases sampling the inner core along the equatorial paths (whose ray angles are greater than $35^{\circ}$ from the Earth's rotation axis). Figure 2 illustrates the ray paths of these core phases. $P K I K P(P K P d f)$ is the $P$ wave transmitted through the inner core. $P K i K P(P K P c d)$ is the $P$ wave reflected off the inner core boundary (ICB). $P K P B_{\text {diff }}$ is the diffracted $P$ wave transmitted through the middle portion of the outer core with a strong focus of energy at the core-mantle boundary (CMB). $P K P b c$ and $P K P a b$ are the $P$ waves propagating through the bottom and middle portions of the outer core, respectively. $P K P B_{\mathrm{diff}}, P K i K P$, and $P K I K P$ can be observed in the long-period seismograms at the $P K P$ caustics distance range $141^{\circ}-145^{\circ}$. The core phases at other distance ranges

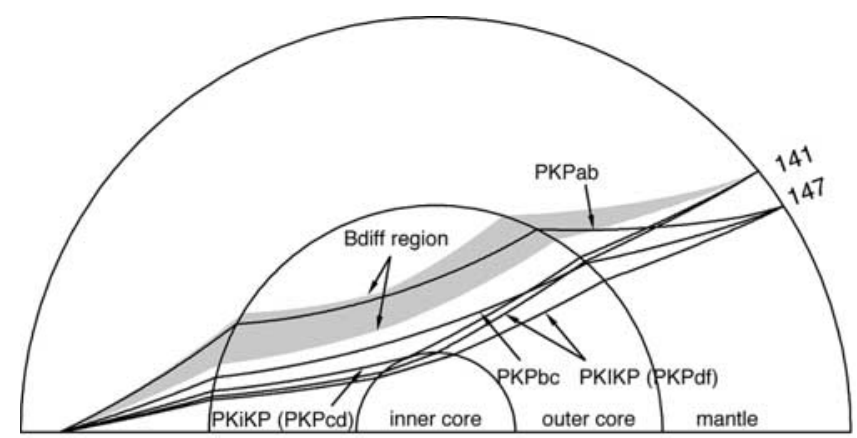

Figure 2. Ray paths of various $P K P$ branches based on PREM: $P K P B_{\text {diff }}\left(B_{\text {diff }}\right), P K i K P(P K P c d), P K I K P(P K P d f)$ at $141^{\circ}$ and PKPab, PKPbc, PKIKP (PKPdf) at $147^{\circ}$. $P K P B_{\text {diff }}$ is the diffracted $P K P$ wave with strong focusing caustics at the core-mantle boundary (CMB) (the shaded area indicates its diffracted region). Note that the separations between the PKiKP and PKIKP paths and the PKPbc and $P K I K P$ paths are small in the mantle, while those between the $P K i K P$ and $P K P B_{\text {diff }}$ paths and the $P K P a b$ and $P K P b c$ paths are large. 
Table 1. Event List

\begin{tabular}{|c|c|c|c|c|c|}
\hline \multirow[b]{2}{*}{ Event } & \multicolumn{2}{|c|}{ Origin Time } & \multirow[b]{2}{*}{ Latitude, ${ }^{\circ} \mathrm{N}$} & \multirow[b]{2}{*}{ Longitude, ${ }^{\circ} \mathrm{E}$} & \multirow[b]{2}{*}{ Depth, km } \\
\hline & Date, year/month/day & Time, UT & & & \\
\hline 19900104 & $1990 / 1 / 4$ & $0532: 25$ & -15.05 & -172.90 & 83 \\
\hline 19900225 & $1990 / 2 / 25$ & 2251:08 & -18.11 & -69.30 & 141 \\
\hline 19900227 & $1990 / 2 / 27$ & 0928:00 & -17.13 & -64.22 & 611 \\
\hline 19900520 & $1990 / 5 / 20$ & 0953:47 & -32.42 & 179.60 & 346 \\
\hline 19901104 & $1990 / 11 / 4$ & 1813:42 & -15.70 & -72.70 & 114 \\
\hline 19910815 & $1991 / 8 / 15$ & 1336:01 & -16.01 & 167.92 & 188 \\
\hline 19920228 & $1992 / 2 / 28$ & 0914:00 & -24.27 & -69.91 & 57 \\
\hline 19920824 & $1992 / 8 / 24$ & 1940:35 & -56.60 & -26.56 & 109 \\
\hline 19930209 & $1993 / 2 / 9$ & $1425: 38$ & 45.69 & 141.93 & 306 \\
\hline 19930224 & $1993 / 2 / 24$ & $2221: 37$ & -24.90 & -68.38 & 117 \\
\hline 19930420 & $1993 / 4 / 20$ & $1626: 19$ & -20.76 & -178.72 & 592 \\
\hline 19930506 & $1993 / 5 / 6$ & 1303:19 & -8.47 & -71.49 & 586 \\
\hline 19930512 & $1993 / 5 / 12$ & 1159:14 & -20.41 & -177.90 & 533 \\
\hline 19930524 & $1993 / 5 / 24$ & 2351:00 & -23.22 & -66.64 & 238 \\
\hline 19930527 & $1993 / 5 / 27$ & 0851:59 & -29.40 & -178.26 & 121 \\
\hline 19930530 & $1993 / 5 / 30$ & $1632: 28$ & -5.48 & 150.49 & 110 \\
\hline 19930709 & $1993 / 7 / 9$ & $1537: 55$ & -19.79 & -177.54 & 412 \\
\hline 19930826 & $1993 / 8 / 26$ & 0332:42 & -5.49 & 154.21 & 135 \\
\hline 19930904 & $1993 / 9 / 4$ & 0830:56 & -16.11 & -176.76 & 389 \\
\hline 19930929 & $1993 / 9 / 29$ & 1903:07 & -6.07 & 149.49 & 63 \\
\hline 19931008 & $1993 / 10 / 8$ & $1823: 46$ & 46.49 & 150.02 & 163 \\
\hline 19931019 & $1993 / 10 / 19$ & $0402: 22$ & -22.39 & -66.00 & 278 \\
\hline 19931030 & $1993 / 10 / 30$ & 1759:02 & -31.75 & -68.21 & 109 \\
\hline 19931110 & 1993/11/10 & 0003:25 & -4.68 & 151.91 & 113 \\
\hline 19940105 & $1994 / 1 / 5$ & 0424:00 & 16.66 & 145.60 & 592 \\
\hline 19940109 & $1994 / 1 / 9$ & 2129:02 & 48.50 & 154.50 & 66 \\
\hline 19940211 & $1994 / 2 / 11$ & $2117: 32$ & -18.81 & 169.16 & 204 \\
\hline 19940420 & $1994 / 4 / 20$ & 0259:11 & -15.03 & -70.53 & 208 \\
\hline 19940510 & $1994 / 5 / 10$ & 0149:03 & -19.72 & -69.85 & 53 \\
\hline 19940522 & $1994 / 5 / 22$ & 0257:15 & -24.26 & -66.91 & 192 \\
\hline 19940705 & $1994 / 7 / 5$ & 0259:42 & -16.33 & -177.50 & 413 \\
\hline 19940813 & $1994 / 8 / 13$ & 2207:09 & 15.13 & 145.87 & 87 \\
\hline 19940816 & $1994 / 8 / 16$ & 1009:34 & 37.83 & 142.46 & 34 \\
\hline 19940819 & $1994 / 8 / 19$ & $1002: 51$ & -26.65 & -63.38 & 565 \\
\hline 19940831 & $1994 / 8 / 31$ & 0907:26 & 43.70 & 145.99 & 80 \\
\hline 19940930 & $1994 / 9 / 30$ & $1930: 16$ & -21.06 & -179.25 & 613 \\
\hline 19941011 & 1994/10/11 & 0137:00 & -32.11 & -71.44 & 51 \\
\hline 19941020 & $1994 / 10 / 20$ & 0115:16 & -39.19 & -70.80 & 164 \\
\hline 19941109 & $1994 / 11 / 9$ & 1821:03 & 43.52 & 147.19 & 60 \\
\hline 19941124 & $1994 / 11 / 24$ & $1321: 15$ & -5.33 & 150.49 & 142 \\
\hline 19941212 & $1994 / 12 / 12$ & 0741:55 & -17.50 & -69.65 & 151 \\
\hline 19941218 & $1994 / 12 / 18$ & 2038:32 & -17.86 & -178.69 & 551 \\
\hline 19941227 & $1994 / 12 / 27$ & $1732: 52$ & -32.00 & 179.87 & 228 \\
\hline 19950120 & $1995 / 1 / 20$ & 0335:46 & 43.26 & 146.82 & 60 \\
\hline 19950121 & $1995 / 1 / 21$ & 0847:29 & 43.34 & 146.72 & 62 \\
\hline 19950206 & $1995 / 2 / 6$ & $1351: 35$ & 41.12 & 142.19 & 70 \\
\hline 19950218 & $1995 / 2 / 18$ & 1329:06 & 46.67 & 145.89 & 354 \\
\hline 19950310 & $1995 / 3 / 10$ & $0522: 22$ & 46.08 & 143.54 & 350 \\
\hline 19950312 & $1995 / 3 / 12$ & $1209: 43$ & -5.33 & 146.70 & 233 \\
\hline 19950316 & $1995 / 3 / 16$ & 0434:44 & -21.62 & -176.50 & 182 \\
\hline 19950331 & $1995 / 3 / 31$ & 1401:40 & 38.15 & 135.06 & 364 \\
\hline 19950408 & $1995 / 4 / 8$ & $1745: 18$ & 21.80 & 142.63 & 318 \\
\hline 19950502 & $1995 / 5 / 2$ & 0354:08 & 43.26 & 147.35 & 50 \\
\hline 19950518 & $1995 / 5 / 18$ & 1431:14 & 44.32 & 147.58 & 103 \\
\hline 19950525 & $1995 / 5 / 25$ & 0459:51 & 43.91 & 147.37 & 76 \\
\hline 19950616 & $1995 / 6 / 16$ & 1349:49 & -18.24 & -178.04 & 567 \\
\hline 19950629 & $1995 / 6 / 29$ & 1224:04 & -19.46 & 169.24 & 144 \\
\hline 19950707 & $1995 / 7 / 7$ & $2115: 18$ & 33.95 & 137.12 & 323 \\
\hline 19950715 & $1995 / 7 / 15$ & 0135:14 & -19.86 & -177.61 & 358 \\
\hline 19950726 & $1995 / 7 / 26$ & 0909:50 & -16.17 & -175.05 & 235 \\
\hline 19950803 & $1995 / 8 / 3$ & 0818:53 & -28.35 & -69.20 & 104 \\
\hline 19950814 & $1995 / 8 / 14$ & $0437: 17$ & -4.83 & 151.51 & 126 \\
\hline 19950818 & $1995 / 8 / 18$ & 0157:18 & 13.21 & 145.15 & 71 \\
\hline 19950823 & $1995 / 8 / 23$ & 0706:02 & 18.86 & 145.19 & 596 \\
\hline 19950824 & $1995 / 8 / 24$ & 0155:34 & 18.92 & 144.95 & 588 \\
\hline 19950912 & $1995 / 9 / 12$ & $1423: 33$ & -21.60 & -179.43 & 599 \\
\hline 19950914 & $1995 / 9 / 14$ & $1224: 34$ & -17.58 & -179.01 & 533 \\
\hline 19950918 & $1995 / 9 / 18$ & 2022:14 & -20.55 & -178.68 & 617 \\
\hline 19950919 & $1995 / 9 / 19$ & 0331:00 & -21.23 & -68.74 & 110 \\
\hline 19950923 & $1995 / 9 / 23$ & $2231: 58$ & -10.53 & -78.70 & 73 \\
\hline 19951014 & $1995 / 10 / 14$ & 0800:41 & -25.57 & -177.51 & 70 \\
\hline
\end{tabular}


Table 1. (continued)

\begin{tabular}{|c|c|c|c|c|c|}
\hline \multirow[b]{2}{*}{ Event } & \multicolumn{2}{|c|}{ Origin Time } & \multirow[b]{2}{*}{ Latitude, ${ }^{\circ} \mathrm{N}$} & \multirow[b]{2}{*}{ Longitude, ${ }^{\circ} \mathrm{E}$} & \multirow[b]{2}{*}{ Depth, km } \\
\hline & Date, year/month/day & Time, UT & & & \\
\hline 19951023 & $1995 / 10 / 23$ & 0358:00 & -14.21 & 167.22 & 211 \\
\hline 19951029 & $1995 / 10 / 29$ & $1940: 57$ & -21.70 & -179.48 & 611 \\
\hline 19951129 & $1995 / 11 / 29$ & $1840: 34$ & -16.78 & -176.38 & 335 \\
\hline 19951130 & $1995 / 11 / 30$ & 1509:00 & 44.32 & 145.51 & 127 \\
\hline 19960114 & $1996 / 1 / 14$ & 1324:07 & -26.84 & -177.54 & 112 \\
\hline 19960219 & $1996 / 2 / 19$ & 2328:06 & -22.04 & -179.53 & 608 \\
\hline 19960306 & $1996 / 3 / 6$ & 0135:03 & -18.66 & -174.86 & 134 \\
\hline 19960317 & 1996/3/17 & 1448:00 & -14.70 & 167.30 & 164 \\
\hline 19960420 & $1996 / 4 / 20$ & $2303: 29$ & -22.29 & -179.69 & 593 \\
\hline 19960514 & $1996 / 5 / 14$ & $1236: 59$ & -17.95 & -178.55 & 605 \\
\hline 19960526 & $1996 / 5 / 26$ & 0143:00 & -22.19 & 171.48 & 108 \\
\hline 19960609 & $1996 / 6 / 9$ & 0112:16 & 17.44 & 145.46 & 149 \\
\hline 19960613 & 1996/6/13 & $0657: 58$ & -20.42 & -178.31 & 535 \\
\hline 19960626 & $1996 / 6 / 26$ & 0355:09 & 27.67 & 139.53 & 471 \\
\hline 19960813 & $1996 / 8 / 13$ & 0941:21 & -21.60 & 170.35 & 110 \\
\hline 19960827 & $1996 / 8 / 27$ & 0624:07 & -22.57 & -179.79 & 575 \\
\hline 19960831 & 1996/8/31 & $1558: 29$ & -14.93 & 167.33 & 126 \\
\hline 19960908 & $1996 / 9 / 8$ & 1142:51 & -19.72 & -179.10 & 600 \\
\hline 19960924 & $1996 / 9 / 24$ & 1142:00 & 15.19 & -61.44 & 147 \\
\hline 19961019 & $1996 / 10 / 19$ & 1453:00 & -20.41 & -178.51 & 591 \\
\hline 19961025 & $1996 / 10 / 25$ & 1959:00 & -17.38 & -69.99 & 116 \\
\hline 19961101 & 1996/11/1 & 0332:34 & -1.30 & 149.52 & 33 \\
\hline 19961111 & 1996/11/11 & 0047:21 & -32.54 & -179.05 & 33 \\
\hline 19961114 & $1996 / 11 / 14$ & 0758:58 & -11.38 & 166.46 & 109 \\
\hline 19961114 & $1996 / 11 / 14$ & $1347: 38$ & -21.24 & -176.62 & 192 \\
\hline 19961117 & $1996 / 11 / 17$ & 2111:20 & -22.20 & -179.70 & 592 \\
\hline 19961130 & $1996 / 11 / 30$ & 2214:31 & -18.41 & -177.77 & 600 \\
\hline 19961222 & $1996 / 12 / 22$ & $1453: 27$ & 43.21 & 138.92 & 227 \\
\hline 19970103 & 1997/1/3 & 0358:22 & -19.22 & -174.84 & 140 \\
\hline 19970311 & 1997/3/11 & 0313:59 & -21.13 & -178.86 & 553 \\
\hline 19970321 & $1997 / 3 / 21$ & $1207: 17$ & -31.16 & 179.62 & 449 \\
\hline 19970325 & $1997 / 3 / 25$ & $1644: 32$ & -9.06 & -71.29 & 603 \\
\hline 19970401 & 1997/4/1 & $1833: 32$ & -18.30 & -69.53 & 114 \\
\hline 19970405 & $1997 / 4 / 5$ & $1223: 30$ & -6.49 & 147.41 & 69 \\
\hline 19970412 & $1997 / 4 / 12$ & 0921:56 & -28.17 & -178.37 & 184 \\
\hline 19970508 & 1997/5/8 & 0556:10 & -19.43 & -175.83 & 218 \\
\hline 19970517 & 1997/5/17 & 0210:18 & -27.16 & -69.50 & 106 \\
\hline 19970527 & $1997 / 5 / 27$ & 1509:00 & 16.33 & 145.44 & 536 \\
\hline 19970611 & 1997/6/11 & 0929:23 & -23.97 & -177.51 & 164 \\
\hline 19970825 & $1997 / 8 / 25$ & 1159:00 & -20.80 & -177.78 & 394 \\
\hline 19970826 & $1997 / 8 / 26$ & 1522:09 & -25.51 & 178.33 & 610 \\
\hline 19970904 & 1997/9/4 & 0423:37 & -26.57 & 178.34 & 625 \\
\hline 19970928 & $1997 / 9 / 28$ & $2313: 13$ & -22.41 & -68.45 & 107 \\
\hline 19971003 & $1997 / 10 / 3$ & 0015:00 & -6.29 & 148.49 & 100 \\
\hline 19971005 & $1997 / 10 / 5$ & 1804:30 & -59.74 & -29.20 & 274 \\
\hline 19971008 & $1997 / 10 / 8$ & $1047: 49$ & -29.25 & 178.35 & 617 \\
\hline 19971115 & 1997/11/15 & 0705:16 & 43.81 & 145.02 & 161 \\
\hline 19971118 & 1997/11/18 & $1541: 29$ & -29.06 & -177.65 & 52 \\
\hline 19971128 & $1997 / 11 / 28$ & 0610:47 & 47.14 & 145.60 & 394 \\
\hline 19971128 & $1997 / 11 / 28$ & 2253:41 & -13.74 & -68.79 & 586 \\
\hline 19971211 & $1997 / 12 / 11$ & $0756: 28$ & 3.93 & -75.79 & 178 \\
\hline 19971216 & $1997 / 12 / 16$ & $2350: 45$ & -5.87 & 147.17 & 103 \\
\hline 19971220 & $1997 / 12 / 20$ & $1326: 31$ & 53.42 & 152.76 & 614 \\
\hline 19980127 & $1998 / 1 / 27$ & 0214:12 & -20.77 & -179.18 & 643 \\
\hline 19980325 & $1998 / 3 / 25$ & 2102:55 & -24.34 & -66.99 & 197 \\
\hline 19980329 & $1998 / 3 / 29$ & 1948:16 & -17.55 & -179.09 & 537 \\
\hline 19980516 & $1998 / 5 / 16$ & 0222:03 & -22.23 & -179.52 & 586 \\
\hline 19980607 & 1998/6/7 & $1610: 46$ & -31.52 & -67.83 & 113 \\
\hline 19980612 & $1998 / 6 / 12$ & 2051:01 & -24.80 & 179.83 & 502 \\
\hline 19980612 & 1998/6/12 & 2153:00 & -5.72 & 147.89 & 140 \\
\hline 19980814 & 1998/8/14 & 0055:37 & -11.43 & 166.22 & 58 \\
\hline 19980824 & $1998 / 8 / 24$ & 0245:34 & -31.89 & -69.45 & 115 \\
\hline 19980901 & 1998/9/1 & $1029: 49$ & -58.21 & -26.53 & 152 \\
\hline 19980902 & 1998/9/2 & 1852:42 & -29.69 & -178.79 & 230 \\
\hline 19980912 & $1998 / 9 / 12$ & 0903:48 & -24.51 & -67.12 & 187 \\
\hline 19980912 & 1998/9/12 & 1058:04 & -14.23 & -72.61 & 91 \\
\hline 19980927 & $1998 / 9 / 27$ & $1107: 16$ & -20.27 & -175.88 & 207 \\
\hline 19981008 & 1998/10/8 & 0451:42 & -16.12 & -71.40 & 136 \\
\hline 19981011 & 1998/10/11 & $1204: 54$ & -21.04 & -179.11 & 624 \\
\hline 19981026 & $1998 / 10 / 26$ & $1356: 20$ & -4.19 & 142.12 & 124 \\
\hline 19981114 & $1998 / 11 / 14$ & $1503: 12$ & -14.95 & 167.37 & 115 \\
\hline
\end{tabular}


Table 1. (continued)

\begin{tabular}{|c|c|c|c|c|c|}
\hline \multirow[b]{2}{*}{ Event } & \multicolumn{2}{|c|}{ Origin Time } & \multirow[b]{2}{*}{ Latitude, ${ }^{\circ} \mathrm{N}$} & \multirow[b]{2}{*}{ Longitude, ${ }^{\circ} \mathrm{E}$} & \multirow[b]{2}{*}{ Depth, km } \\
\hline & Date, year/month/day & Time, UT & & & \\
\hline 19981124 & $1998 / 11 / 24$ & $2354: 46$ & -16.51 & -174.75 & 223 \\
\hline 19990109 & $1999 / 1 / 9$ & 0305:00 & 44.39 & 147.31 & 119 \\
\hline 19990127 & $1999 / 1 / 27$ & $0337: 47$ & -22.31 & -179.02 & 437 \\
\hline 19990129 & $1999 / 1 / 29$ & $1504: 00$ & -18.73 & 169.25 & 237 \\
\hline 19990302 & $1999 / 3 / 2$ & $1745: 00$ & -22.72 & -68.50 & 111 \\
\hline 19990305 & $1999 / 3 / 5$ & $0033: 48$ & -19.89 & -68.56 & 111 \\
\hline 19990306 & $1999 / 3 / 6$ & 2028:00 & -21.73 & -179.46 & 603 \\
\hline 19990312 & $1999 / 3 / 12$ & 1116:00 & -20.00 & -177.76 & 587 \\
\hline 19990323 & $1999 / 3 / 23$ & 1123:00 & -20.91 & -178.73 & 575 \\
\hline 19990626 & $1999 / 6 / 26$ & $2205: 00$ & -17.96 & -178.19 & 590 \\
\hline 19990629 & $1999 / 6 / 29$ & $2318: 00$ & 36.62 & 71.35 & 189 \\
\hline 19990719 & $1999 / 7 / 19$ & 0217:03 & -28.63 & -177.61 & 39 \\
\hline 19990721 & $1999 / 7 / 21$ & 0310:44 & -18.29 & -177.91 & 561 \\
\hline 19990818 & $1999 / 8 / 18$ & $0116: 48$ & -37.34 & 177.33 & 155 \\
\hline 19990822 & $1999 / 8 / 22$ & 0935:39 & -40.51 & -74.76 & 33 \\
\hline 19990825 & $1999 / 8 / 25$ & 0706:22 & -19.06 & 169.61 & 263 \\
\hline 19990828 & $1999 / 8 / 28$ & $1240: 06$ & -1.29 & -77.55 & 196 \\
\hline 19990915 & $1999 / 9 / 15$ & 0301:00 & -20.93 & -67.28 & 218 \\
\hline 19991121 & $1999 / 11 / 21$ & $0351: 14$ & -21.75 & -68.78 & 101 \\
\hline 19991127 & $1999 / 11 / 27$ & 0231:49 & -14.53 & -71.29 & 126 \\
\hline 19991130 & $1999 / 11 / 30$ & 2010:00 & -21.33 & -178.66 & 548 \\
\hline 19991210 & $1999 / 12 / 10$ & 0334:45 & -22.30 & 179.61 & 600 \\
\hline 20000102 & $2000 / 1 / 2$ & $1214: 39$ & -17.94 & -178.48 & 582 \\
\hline 20000113 & $2000 / 1 / 13$ & $2007: 14$ & -17.61 & -178.74 & 535 \\
\hline 20000115 & $2000 / 1 / 15$ & $1249: 45$ & -21.22 & -179.26 & 633 \\
\hline 20000213 & $2000 / 2 / 13$ & 0257:08 & 42.85 & 131.57 & 514 \\
\hline 20000215 & $2000 / 2 / 15$ & 0205:00 & 17.67 & 145.40 & 522 \\
\hline 20000226 & $2000 / 2 / 26$ & 0811:00 & 13.80 & 144.78 & 132 \\
\hline 20000228 & $2000 / 2 / 28$ & $2215: 20$ & -17.59 & -178.98 & 538 \\
\hline 20000301 & $2000 / 3 / 1$ & 0421:01 & -19.00 & -179.36 & 675 \\
\hline 20000315 & $2000 / 3 / 15$ & 0635:05 & -44.40 & -117.43 & 10 \\
\hline 20000318 & $2000 / 3 / 18$ & $2322: 00$ & -24.36 & 178.98 & 552 \\
\hline 20000322 & $2000 / 3 / 22$ & $2332: 26$ & -14.56 & 167.27 & 169 \\
\hline 20000401 & $2000 / 4 / 1$ & $1210: 49$ & -17.90 & -178.65 & 608 \\
\hline 20000401 & $2000 / 4 / 1$ & $1213: 24$ & -17.76 & -178.75 & 556 \\
\hline 20000407 & $2000 / 4 / 7$ & $1842: 00$ & -18.27 & -175.27 & 208 \\
\hline 20000411 & $2000 / 4 / 11$ & $0641: 26$ & -27.94 & -178.39 & 201 \\
\hline 20000417 & $2000 / 4 / 17$ & 0425:00 & -22.17 & -179.35 & 539 \\
\hline 20000418 & $2000 / 4 / 18$ & $1728: 12$ & -20.66 & -176.47 & 221 \\
\hline 20000423 & $2000 / 4 / 23$ & $0927: 23$ & -28.31 & -62.99 & 609 \\
\hline 20000423 & $2000 / 4 / 23$ & $1701: 17$ & -28.38 & -62.94 & 610 \\
\hline 20000429 & $2000 / 4 / 29$ & $1952: 21$ & -6.41 & -77.06 & 125 \\
\hline 20000504 & $2000 / 5 / 4$ & $2036: 32$ & -17.91 & -178.52 & 516 \\
\hline 20000508 & $2000 / 5 / 8$ & $1028: 25$ & -4.46 & 150.00 & 502 \\
\hline 20000508 & $2000 / 5 / 8$ & $2135: 42$ & -31.32 & 179.84 & 383 \\
\hline 20000614 & $2000 / 6 / 14$ & 0215:00 & -25.52 & 178.05 & 605 \\
\hline 20000614 & $2000 / 6 / 14$ & 0319:18 & -24.03 & -66.75 & 197 \\
\hline 20000616 & $2000 / 6 / 16$ & $0755: 35$ & -33.88 & -70.09 & 120 \\
\hline 20000710 & $2000 / 7 / 10$ & 0958:00 & 46.83 & 145.42 & 360 \\
\hline 20000802 & $2000 / 8 / 2$ & $0037: 16$ & -17.94 & -174.82 & 191 \\
\hline 20000815 & $2000 / 8 / 15$ & 0430:08 & -31.51 & 179.73 & 358 \\
\hline 20000902 & $2000 / 9 / 2$ & 1019:13 & -17.92 & -178.32 & 588 \\
\hline 20000902 & $2000 / 9 / 2$ & $1702: 19$ & -20.07 & -179.13 & 688 \\
\hline 20000903 & $2000 / 9 / 3$ & $0821: 23$ & -20.55 & -177.82 & 364 \\
\hline 20000911 & $2000 / 9 / 11$ & $1717: 53$ & -15.88 & -173.69 & 115 \\
\hline 20000914 & $2000 / 9 / 14$ & $1459: 57$ & -15.74 & 179.80 & 33 \\
\hline 20000922 & $2000 / 9 / 22$ & $1321: 31$ & -29.85 & -178.38 & 157 \\
\hline 20000926 & $2000 / 9 / 26$ & $0617: 52$ & -17.18 & -173.93 & 56 \\
\hline 20001004 & $2000 / 10 / 4$ & $1437: 44$ & 11.12 & -62.56 & 110 \\
\hline 20001011 & $2000 / 10 / 11$ & 0413:35 & -20.65 & -177.99 & 489 \\
\hline 20001025 & $2000 / 10 / 25$ & 1900:17 & -34.68 & -109.46 & 10 \\
\hline 20001030 & $2000 / 10 / 30$ & 2028:00 & -10.51 & 165.76 & 108 \\
\hline 20001109 & $2000 / 11 / 9$ & $0545: 54$ & -15.43 & -173.42 & 53 \\
\hline 20001113 & $2000 / 11 / 13$ & $2317: 28$ & -21.26 & -179.23 & 628 \\
\hline 20001218 & $2000 / 12 / 18$ & 0119:21 & -21.18 & -179.12 & 628 \\
\hline 20001218 & $2000 / 12 / 18$ & $2115: 30$ & -21.18 & -179.10 & 649 \\
\hline 20001225 & $2000 / 12 / 25$ & $0511: 58$ & -21.26 & -179.12 & 644 \\
\hline
\end{tabular}




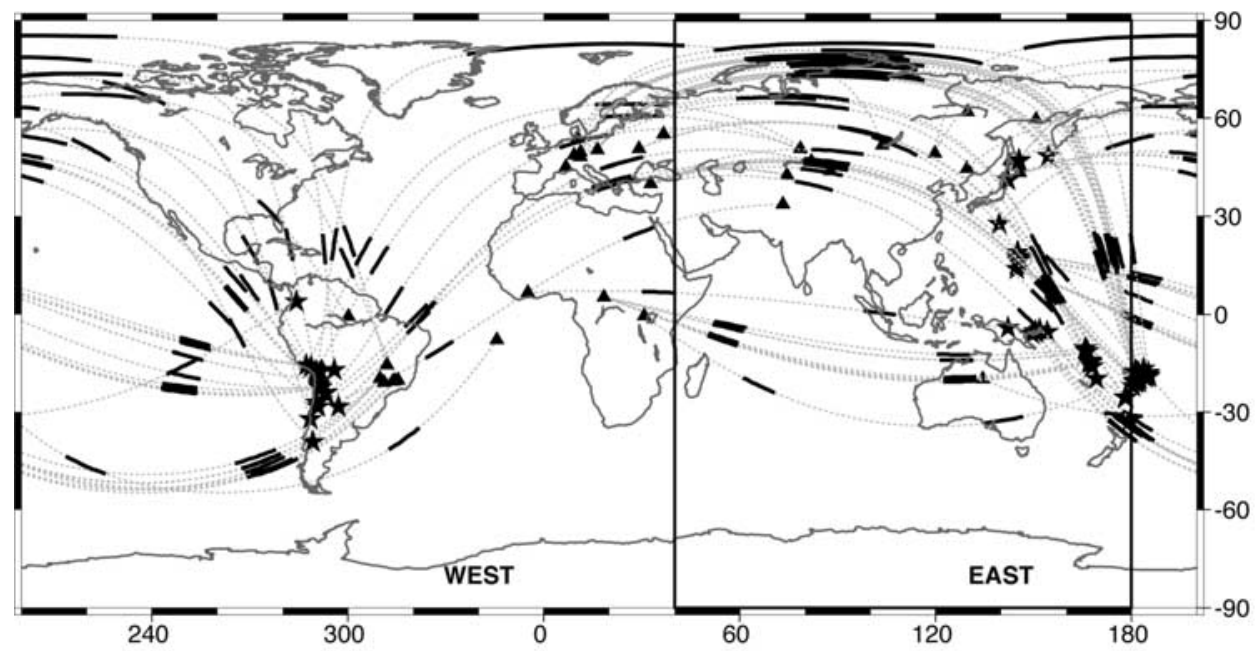

Figure 3. Map view of great circle paths (dotted lines) and their sampling regions at the bottom $200 \mathrm{~km}$ of the outer core (heavy segments) of the PKIKP phases used in the analyses of PKPbc-PKIKP travel times and waveforms. The seismic data are collected from the stations in the Global Seismographic Network (GSN) and several regional arrays in South America (The Broadband Andean Joint Experiment, BANJO, and BLSP), Japan (FREESIA), and German (Grafenberg, GRF, and German Regional Seismic Network, GRSN). Stars and triangles indicate the locations of earthquakes and stations, respectively. The geographic separation of the two "hemispheres" is also marked. The "eastern" hemisphere is defined between $40^{\circ} \mathrm{E}$ and $180^{\circ} \mathrm{E}$, and the "western" hemisphere is defined between $180^{\circ} \mathrm{W}$ and $40^{\circ} \mathrm{E}$.

can be easily identified in the short-period seismograms. The differential travel times and waveforms of these core phase pairs are sensitive to the seismic structures in different parts of the Earth in different ways. PKiKP-PKIKP waveforms and differential travel times are sensitive to the seismic structures in the top $80 \mathrm{~km}$ of the inner core and in the bottom of the outer core [Wen and Niu, 2002]. $P K i K P-P K P B_{\text {diff }}$ differential travel times are sensitive to the seismic structure at the bottom of the outer core and the long-wavelength seismic structure near the CMB. $P K P b c$ $P K I K P$ differential travel times are most sensitive to the seismic structures in the bottom of the outer core and in the top of the inner core. PKPab-PKPbc differential travel times are sensitive to the velocity structures near the $\mathrm{CMB}$ and the ICB. $P K P b c / P K P a b$ amplitude ratios at larger distances can be used to further constrain the seismic velocity structure at the bottom of the outer core when the $P K P b c$ waves diffract along the ICB.

[5] We have assembled a vast number of observations of the PKP phases from the seismic data recorded in the IRIS Global Seismographic Network (GSN) from 1990 to 2000 and in several dense regional seismic networks: Grafenberg (GRF) and German Regional Seismic Network (GRSN) in German, the Broadband Andean Joint Experiment (BANJO) and BLSP in South America, and FREESIA in Japan (see earthquake parameters in Table 1). We divide the data into three categories according to the geographic sampling by the PKIKP phases: "east", "west", and "east-west transition". The east and west categories are defined when both PKIKP entry and exit points are within the "eastern" $\left(40^{\circ} \mathrm{E}-180^{\circ} \mathrm{E}\right)$ and "western" hemispheres $\left(180^{\circ} \mathrm{W}-40^{\circ} \mathrm{E}\right)$ of the inner core, respectively. The "east-west transition" is defined by other cases, i.e., PKIKP entry and exit points are in different "hemispheres". Every event is checked for the simplicity of its source time function. Every seismogram is checked by eye for its quality. Short-/long-period seismograms are broadband seismograms filtered with the World-Wide Standard Seismograph Network (WWSSN) short-/longperiod instrumental response. Our selected data sets include: 260 short-period $P K i K P-P K I K P$ waveforms at $120^{\circ}-141^{\circ}$, 55 long-period $P K i K P-P K P B_{\text {diff }}$ waveforms within the $B$ caustics distance range $\left(141^{\circ}-145^{\circ}\right), 96$ short-period $P K P b c-P K I K P$ waveforms at the distance range of $144^{\circ}-148^{\circ}, 368$ short-period $P K P b c-P K I K P$ waveforms at the distance range of $147^{\circ}-153^{\circ}$, and 406 short-period $P K P a b-P K P b c$ waveforms at the distance range of $146^{\circ}-159^{\circ}$. These data sets were selected from the collections of more than 16000 observations. The $P K i K P-P K I K P$ waveforms at the distance range of $120^{\circ}-141^{\circ}$ were collected in the previous study by Wen and Niu [2002]. Other data sets are new collections in this study.

\subsection{Seismic Observations of the PKPbc-PKIKP Phases at $144^{\circ}-148^{\circ}$}

[6] We begin by presenting the differential travel times and waveforms of the short-period PKPbc-PKIKP phases observed at the distance range of $144^{\circ}-148^{\circ}$ along the equatorial paths. The geographic coverage of the bottom $200 \mathrm{~km}$ of the outer core sampled by the PKIKP phases is restricted by event locations, simplicity of source time function, and quality of the data (Figure 3). The $P K P b c-$ $P K I K P$ differential travel times are determined by measuring the time difference between the handpicked maximum amplitudes of the two phases. The difference between the differential travel times determined by the handpicked maximum amplitudes and those determined by the method 
of cross correlation of the two phases is less than $0.05 \mathrm{~s}$. $P K P b c-P K I K P$ waveforms and differential travel times exhibit a clear "hemispheric" difference (Figures 4 and 5). PKIKP phases bifurcate at a closer epicentral distance and arrive about $0.7 \mathrm{~s}$ earlier for those sampling the "eastern" hemisphere than those sampling the "western" hemisphere. The development of the PKPbc-PKIKP waveforms near the PKP caustics distance can also be used to place constraints on the detailed seismic properties near the ICB. We expand short-period PKP waveform collections back to $144^{\circ}$ (Figures 4a and 4d). Observed PKP waveforms are aligned along the maximum amplitudes of the $P K P b c$ phases. Distance corrections are made so that each trace is aligned equivalently to a common source depth of a) EAST (data)

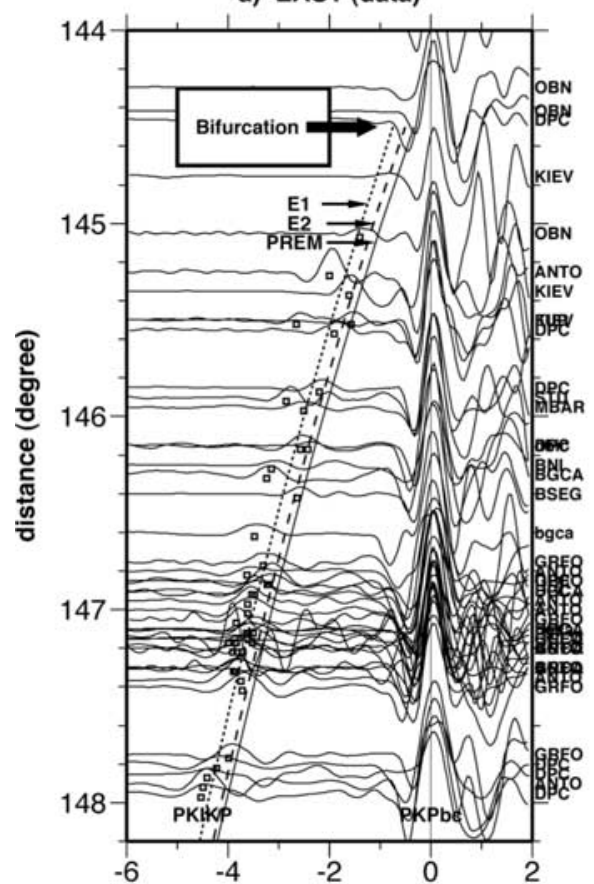

d) WEST (data)

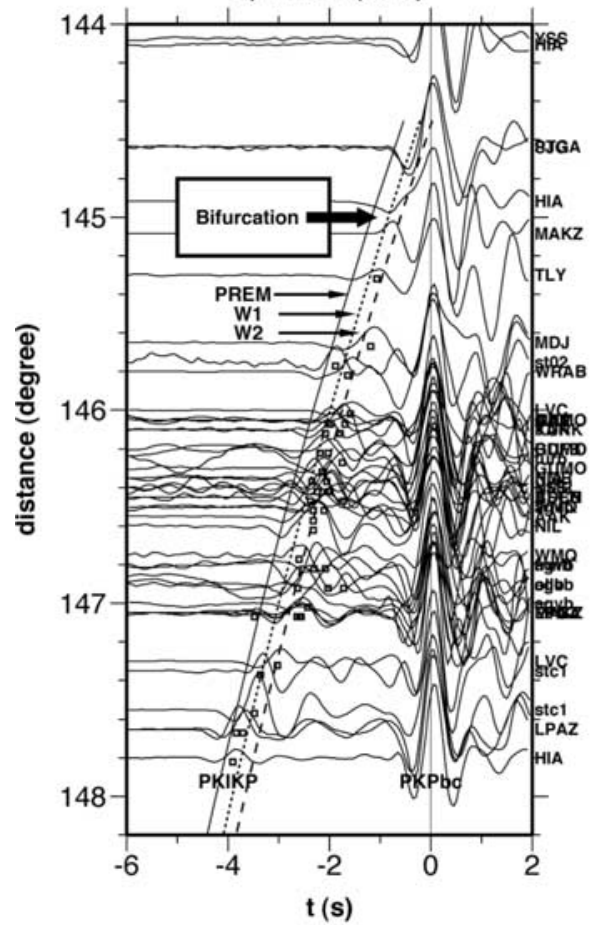

b) E1 (synthetics)

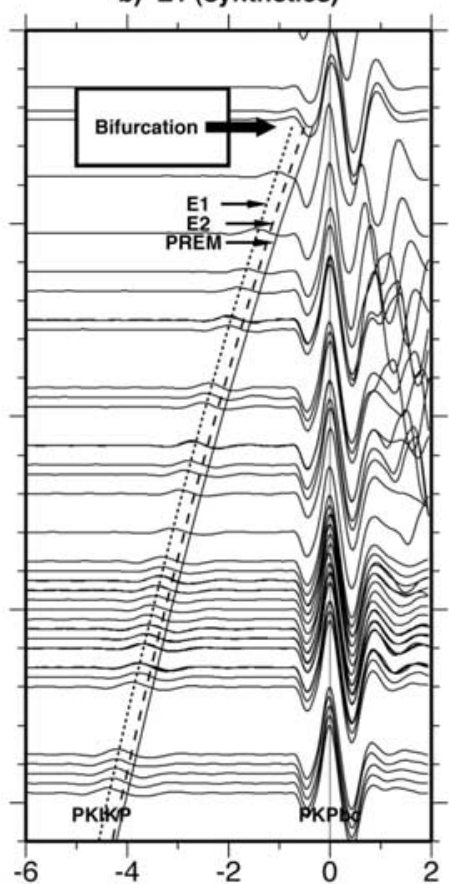

e) W1 (synthetics)

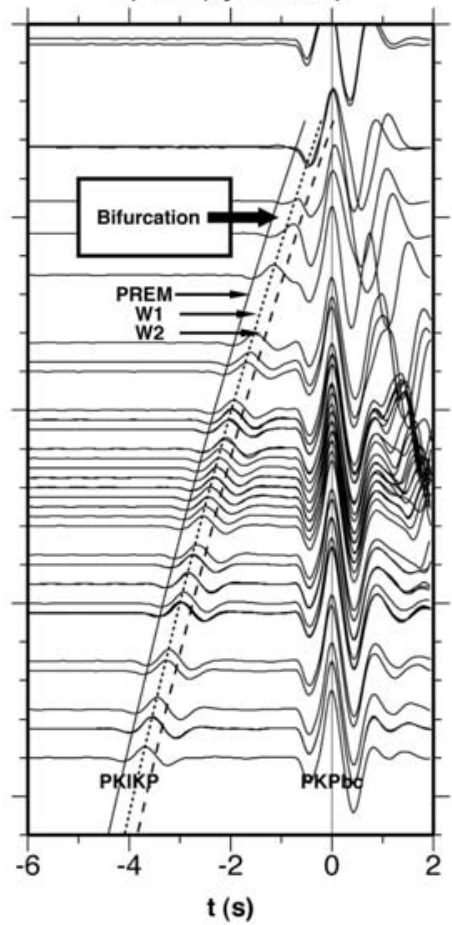

Figure 4 c) E2 (synthetics)

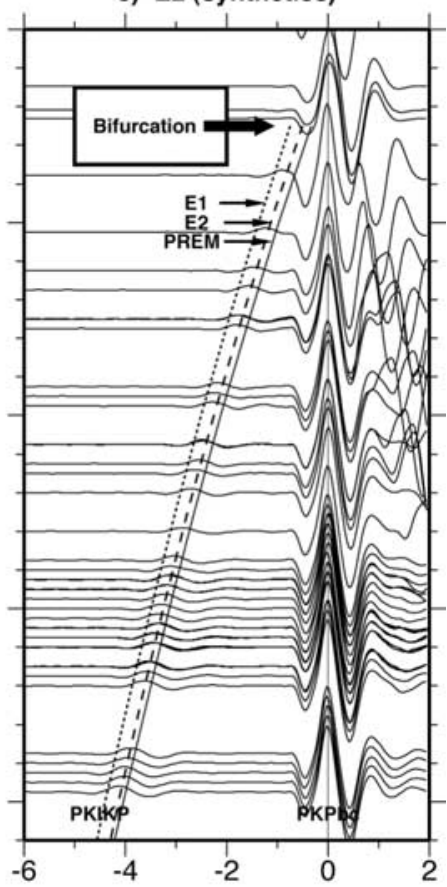

f) W2 (synthetics)

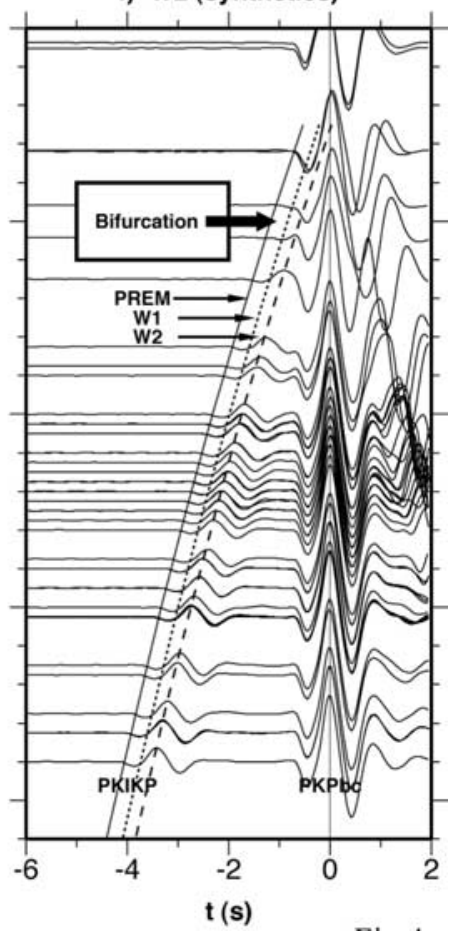


$600 \mathrm{~km}$. PKIKP phases bifurcate near $144.5^{\circ}$ for the seismic data sampling the eastern hemisphere (Figure 4a), and near $144.9^{\circ}$ for those sampling the western hemisphere (Figure 4d).

[7] At the distance range of $144^{\circ}-148^{\circ}, P K P b c$ waves turn at the outer core in a region $500-240 \mathrm{~km}$ above the ICB, whereas PKIKP waves propagate through the outer core and the top $140-180 \mathrm{~km}$ of the inner core. Because of the closeness of $P K P b c$ and $P K I K P$ ray paths in the mantle, the $P K P b c-P K I K P$ differential travel times are insensitive to the seismic heterogeneities in the mantle. However, in order to study the seismic structure in the bottom of the outer core, additional constraints on the seismic structure in the top of the inner core are required, as the PKPbc-PKIKP differential travel times would be affected by the seismic structures in both the bottom of the outer core and the top $180 \mathrm{~km}$ of the inner core. This can be achieved by jointly modeling the PKiKP-PKIKP waveforms and the $P K P b c-P K I K P$ differential travel times and waveforms, since the PKiKP-PKIKP observations are indicative of velocity variations in the uppermost portion of the inner core [Kaneshima, 1996; Niu and Wen, 2001; Ouzounis and Creager, 2001; Niu and Wen, 2002; Wen and Niu, 2002]. Wen and Niu [2002] showed that in explaining the PKiKP$P K I K P$ observations, there is a trade-off between the seismic velocity structure in the top of the inner core and the seismic velocity structure in the bottom of the outer core. Because the velocity structure in the top of the inner core trades off with the velocity structure in the bottom of the outer core in such a way that a more gradual velocity gradient at the bottom of the outer core requires a lowervelocity structure in the top of the inner core, the velocity structure in the bottom of the outer core and its coupled velocity structure in the top of the inner core can be resolved by jointly modeling the $P K i K P-P K I K P$ and the $P K P b c-$ $P K I K P$ differential travel times and waveforms. A flat velocity gradient at the bottom of the outer core would delay the $P K I K P$ travel time and produce smaller $P K P b c$ $P K I K P$ differential travel time. In jointly modeling both the $P K i K P-P K I K P$ and the $P K P b c-P K I K P$ data sets, we first fix the velocity structure at the bottom of the outer core to be PREM values and search the velocity jumps across the ICB and the velocities in the top of the inner core, so that the modified models can fit the observed bifurcation distances and the subsequent moveouts of the observed PKiKPPKIKP phases for each hemisphere. The resultant models E1 (for the eastern hemisphere) and W1 (for the western hemisphere) are shown in Figure 6, and their PKiKP$P K I K P$ synthetic waveforms are shown in Figure 7 . We then test different velocity gradients at the bottom of the outer core. For each velocity gradient, we again infer two velocity models in the top of the inner core on the basis of waveform fitting the observed PKiKP-PKIKP phases sampling the two hemispheres. We then search for the best coupled outer core-inner core models on the basis of the observed $P K P b c-P K I K P$ differential travel times. Our modeling results suggest that (Figures 4 and 5) the PREM velocity structure at the bottom of the outer core and its coupled inner core model E1 can explain the differential travel times for the $P K P b c-P K I K P$ data sampling the eastern hemisphere, while a lower-velocity gradient model OW at the bottom of the outer core and its coupled velocity model W2 in the top of the inner core better explain the $P K P b c$ PKIKP data sampling the western hemisphere. OW has reduced velocities relative to PREM linearly increasing from $0 \%$ at the bottom $200 \mathrm{~km}$ above the ICB to $-0.35 \%$ at the ICB (Figure 6).

[8] The bifurcation distances and the PKPbc-PKIKP waveforms near the caustics distance can also be better explained by models with two different types of velocity gradient in the bottom of the outer core for the two hemispheres (Figure 4). For the seismic data sampling the eastern hemisphere, the observed bifurcation distance (near $144.5^{\circ}$ ) and the subsequent moveouts of the $P K P b c-P K I K P$ phases are better explained by E1 synthetics (Figure $4 b$ ) than E2 (E2 has a velocity structure of $\mathrm{OW}$ in the bottom of the outer core) synthetics (Figure 4c), while, for the seismic data sampling the western hemisphere, the bifurcation distance (near $144.9^{\circ}$ ) and the subsequent moveouts of the $P K P b c-P K I K P$ phases are better explained by W2 synthetics (Figure 4f) than W1 synthetics (Figure 4e).

[9] PREM velocities in the bottom of the outer core are unable to simultaneously explain both the PKiKP-PKIKP and the $P K P b c-P K I K P$ waveform and travel time data sampling the western hemisphere. We fix the outer core velocity structure to be PREM and test various inner core models. As we mentioned earlier, W1 in the top of the inner core would explain the observed PKiKP-PKIKP data, but it

\footnotetext{
Figure 4. Observed waveforms for the PKIKP and $P K P b c$ phases sampling the (a) eastern and (d) western hemispheres selected from the recordings in the GSN and several regional networks. Synthetic waveforms calculated on the basis of the four coupled outer core-inner core models (b) E1, (c) E2, (e) W1, and (f) W2 along with the predicted PKIKP arrivals based on E1, W1 (dotted lines), E2, W2 (dashed lines), and PREM (solid lines) (see Figure 6 for the models). E1, E2, W1, and W2 are derived from fitting the observed PKiKP-PKIKP waveforms (Figure 7). The seismograms are band-passfiltered with the WWSSN short-period instrumental response and are aligned along the maximum amplitudes of the $P K P b c$ phases. Maximal handpicked PKIKP amplitudes are indicated by open dots. Each synthetic waveform is calculated on the basis of the epicentral distance and event depth associated with each observation. Distance corrections are made to a common source depth of $600 \mathrm{~km}$ for both the observed and synthetic waveforms. The method we apply to the distance correction is based on the differential travel times of $P K P b c-P K I K P$. We plot each seismogram at an epicentral distance that, for a source depth of $600 \mathrm{~km}$, would generate a same differential travel time as its actual event depth and epicentral distance would do, based on E1 (for the eastern hemisphere, Figure 4a) and W1 (for the western hemisphere, Figure 4d). Accordingly, the predicted PKIKP arrivals from the above models are calculated on the basis of a source depth of $600 \mathrm{~km}$. The predicted $P K I K P$ travel times for the seismic data sampling eastern hemisphere are predicted on the basis of a $\mathrm{Q}$ value of 250 in the top of the inner core (Figures $4 \mathrm{a}, 4 \mathrm{~b}$, and $4 \mathrm{c}$ ).
} 

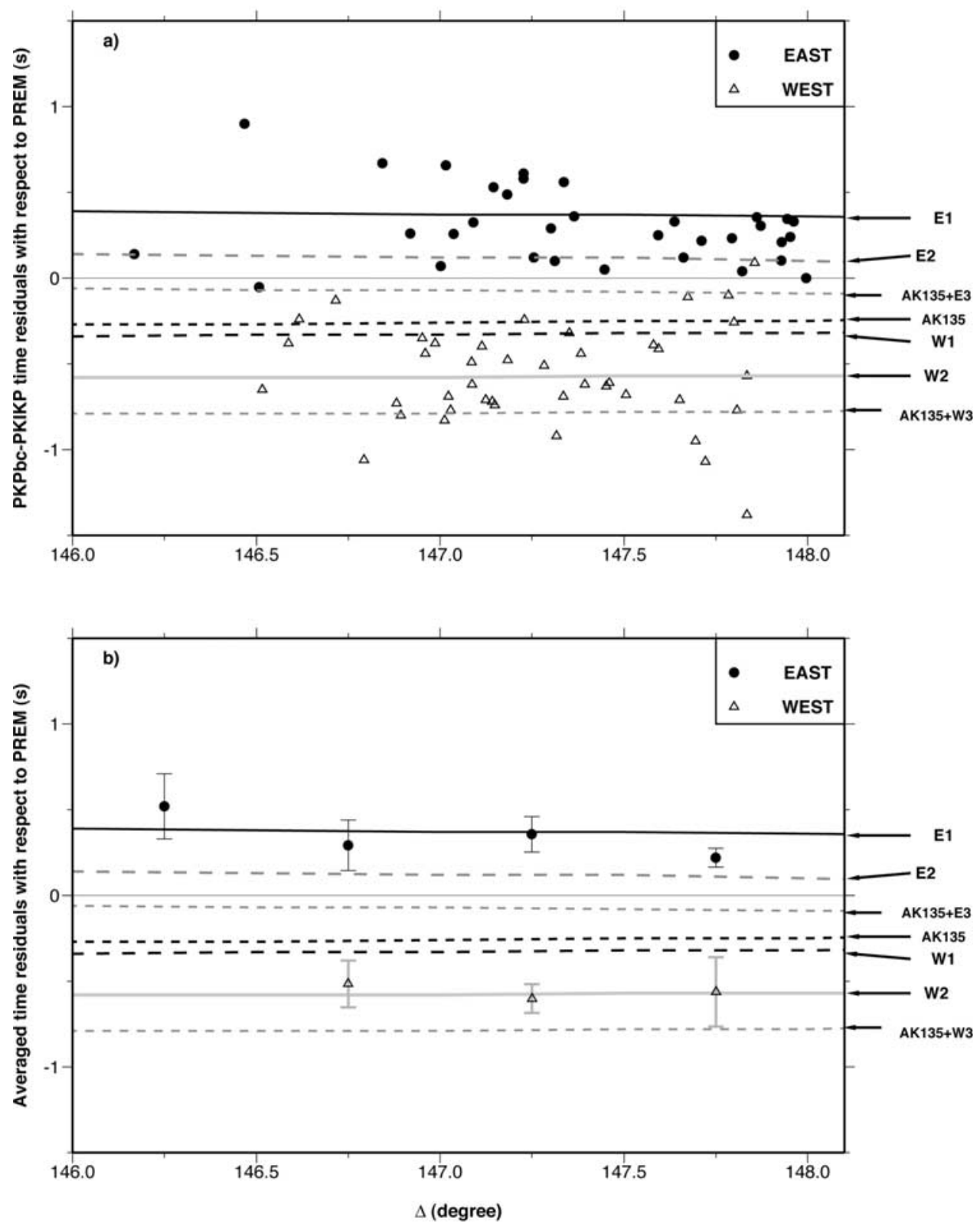

Figure 5. (a) Observed $P K P b c-P K I K P$ differential travel time residuals and (b) their averages over each $0.5^{\circ}$ with respect to PREM plotted as a function of epicentral distance, along with the predictions from E1, W1, E2, W2, AK135, AK135+E3, and AK135+W3 with a common source depth of $600 \mathrm{~km}$. Solid circles and open triangles represent the observed differential travel time residuals for the PKIKP phases sampling the eastern and western hemispheres of the inner core, respectively. Standard deviations are also plotted in Figure 5b. E1 and W2 can better explain the observed PKPbc-PKIKP differential travel time residuals for those sampling the eastern and western hemispheres, respectively.

predicts larger $P K P b c-P K I K P$ travel times than the observations (Figures 7 and 5). If we uniformly lower the W1 velocities in the top of the inner core to fit the $P K P b c$ $P K I K P$ waveforms (let us term this model W13), the predicted $P K i K P$-PKIKP differential travel times would be about $0.3-0.6 \mathrm{~s}$ smaller than most of the PKiKP-PKIKP observations. Any velocity structure between W1 and W13 in the top of the inner core would predict misfits of at least $0.2-0.4 \mathrm{~s}$ to either PKiKP-PKIKP or PKPbc-PKIKP travel times. Those misfits cannot be reconciled by assuming different velocity gradients in the top of the inner core either. We fix the velocity jump across the ICB to be that of $\mathrm{W} 1$, which is required to explain the bifurcation distance and the waveforms observed at the bifurcation distance range for the $P K i K P$-PKIKP data, and change the velocity gradient in the top of the inner core to fit the $P K P b c-P K I K P$ differential travel times. The modified inner core model would predict smaller PKiKP and PKIKP time separations than the observations after $136^{\circ}$. The PKiKP-PKIKP synthetic waveform misfits to the data increase with increasing 


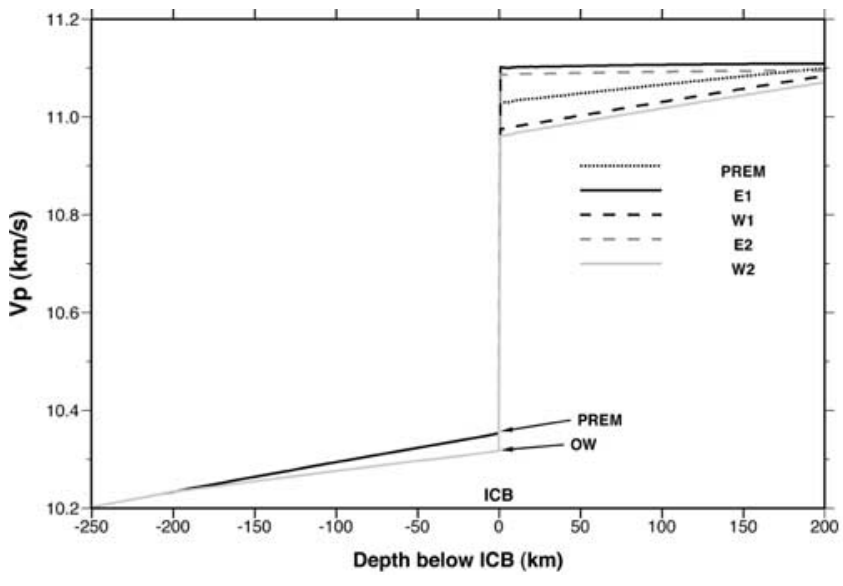

Figure 6. $P$ wave velocity profile near the ICB: PREM, E1, W1, E2, and W2. E1, W1, E2, and W2 are the coupled outer core-inner core models that are capable of explaining the observed PKiKP-PKIKP phases (Figure 7). Initials E and $\mathrm{W}$ stand for models that are appropriate for explaining the data sampling the eastern and western hemispheres, respectively. Numbers 1 and 2 stand for models with PREM and OW structures at the bottom of the outer core, respectively.

epicentral distance. This also confirms that the W1 (W2) velocity gradient in the top of the inner core is well resolved from the PKiKP-PKIKP data, as mentioned by Wen and Niu [2002].

[10] We should emphasize that we have only used the $P K i K P-P K I K P$ and the PKPbc-PKIKP phases whose sampling angles are greater than $35^{\circ}$ from the Earth's rotation axis to avoid the influence from the inner core anisotropy. The inner core anisotropy in velocity exhibits a similar hemispheric pattern between the eastern and western hemispheres, but it has a form that velocity is higher along the polar paths than along the equatorial paths [Tanaka and Hamaguchi, 1997; Creager, 1999; Garcia and Souriau, 2000; Ouzounis and Creager, 2001; Niu and Wen, 2002]. The hemispheric variation in anisotropy has no effect on our inference of seismic velocity structures in the bottom of the outer core, as our outer core structures are inferred on the basis of the data sampling along the equatorial paths in each hemisphere. Our inference of seismic structures in the outer core will only be affected by anisotropy effect in the data within the equatorial path groups (such effect would have increased the $P K P b c-P K I K P$ differential travel times and would require a lower-velocity structure in the bottom of the outer core), if such effect exists. However, the observed $P K P b c-P K I K P$ differential travel times for the seismic data within the equatorial path groups (as we defined) exhibit no dependence on their ray angles for the both hemispheres [e.g., Creager, 1999, Figure 2B], indicating little anisotropy effect in the data within the equatorial path groups in the both hemispheres.

[11] To estimate the possible travel time anomalies caused by the different ray paths of $P K P b c$ and $P K I K P$ in the mantle, we use a $P$ velocity tomographic model by van der Hilst et al. [1997] to correct the $P$ wave travel time delays caused by the seismic heterogeneities in the mantle. The differential travel time residuals change little after the tomographic corrections (Figure 8).

\subsection{Seismic Observations of the $P K i K P-P K P B_{\text {diff }}$ Phases at the $B$ Caustics}

[12] At the $B$ caustics distance range $\left(141^{\circ}-145^{\circ}\right)$, $P K i K P$ and $P K P B_{\text {diff }}$ phases are discernible in the long period seismograms. Figure 9 shows the observed and synthetic $P K i K P-P K P B_{\text {diff }}$ waveforms aligned according to the $P K i K P$ arrivals predicted by PREM. Their differential travel times are useful in constraining seismic structures at the bottom of the outer core and/or at the base of the mantle. However, it is often difficult to unambiguously identify the $P K i K P$ and $P K P B_{\text {diff }}$ phases in the data. As a result, we are only able to select 55 high-quality $P K i K P-P K P B_{\text {diff }}$ waveforms from 2932 seismograms recorded at the GSN, FREESIA, and GRF. Because different earthquakes have different source time functions, measuring the $P K i K P-P K P B_{\text {diff }}$ differential travel time by the time difference between the handpicked maximum amplitudes of the two phases would have a large error. Instead, we measure the travel time residuals on the basis of waveform fitting. For each observed $P K i K P-P K P B_{\text {diff }}$ waveform, we separately calculate the $P K i K P$ and $P K P B_{\text {diff }}$ waveforms on the basis of PREM using the generalized ray theory [Helmberger, 1983]. The synthetics are calculated using the source time function, source depth, and epicentral distance associated with each observation. The measured $P K i K P-P K P B_{\text {diff }}$ time residual is the time shift of the $P K P B_{\text {diff }}$ synthetics when the summation of the $P K i K P$ synthetics and the time-shifted $P K P B_{\text {diff }}$ synthetics fit the data best. The error of measured time residuals is about $0.5 \mathrm{~s}$ due to the long-wavelength characteristic of the $P K P B_{\text {diff }}$ phase. The differential PKiKP$P K P B_{\text {diff }}$ travel time residuals, in general, exhibit a hemispheric pattern as well (Figures 10 and 11). Most of the residuals are negative for the $P K i K P$ phases sampling the eastern hemisphere and positive for those sampling the western hemisphere. The $P K i K P-P K P B_{\text {diff }}$ travel time residuals on average are about $0.9 \mathrm{~s}$ larger for those sampling the western hemisphere than those sampling the eastern hemisphere. These travel time residuals are close to the study by Luo et al. [2002]. A positive/negative residual can be interpreted by a relative low/high $P$ velocity structure at the bottom of the outer core or a relative high/low velocity structure in the lower mantle. For the data sampling the western hemisphere, if we attribute the signals originating in the bottom of the outer core, the observed waveforms can generally be explained by OW. If we attribute the signals to be caused by the seismic structures in the lower mantle, model LM, a model with increased velocities relative to PREM linearly increasing from $0 \%$ at $200 \mathrm{~km}$ above the $\mathrm{CMB}$ to $4 \%$ at the $\mathrm{CMB}$, can generate indistinguishable $P K i K P-P K P B_{\text {diff }}$ synthetic waveforms as $\mathrm{OW}$ does. The observed $P K i K P-P K P B_{\text {diff }}$ waveforms sampling the eastern hemisphere can be explained by PREM in the lower mantle and in the bottom of the outer core. Note that the predicted differential travel time difference between PREM and OW is about $0.3 \mathrm{~s}$, while the observed differential travel times are scattered by more than $1 \mathrm{~s}$ (Figure 11). These observed scatters in the data reflect both uncertainties in extracting the travel time information from the long-period data and 
the effects due to the seismic heterogeneities in the mantle. The large scatters in the travel time make it impossible to use the $P K i K P-P K P B_{\text {diff }}$ data set to resolve the seismic structure in the bottom of the outer core. We again introduce the tomographic model by van der Hilst et al. [1997] to correct the $P K i K P-P K P B_{\text {diff }}$ differential travel time delays caused by the seismic heterogeneities in the mantle. The magnitude of the corrections is larger than that for the
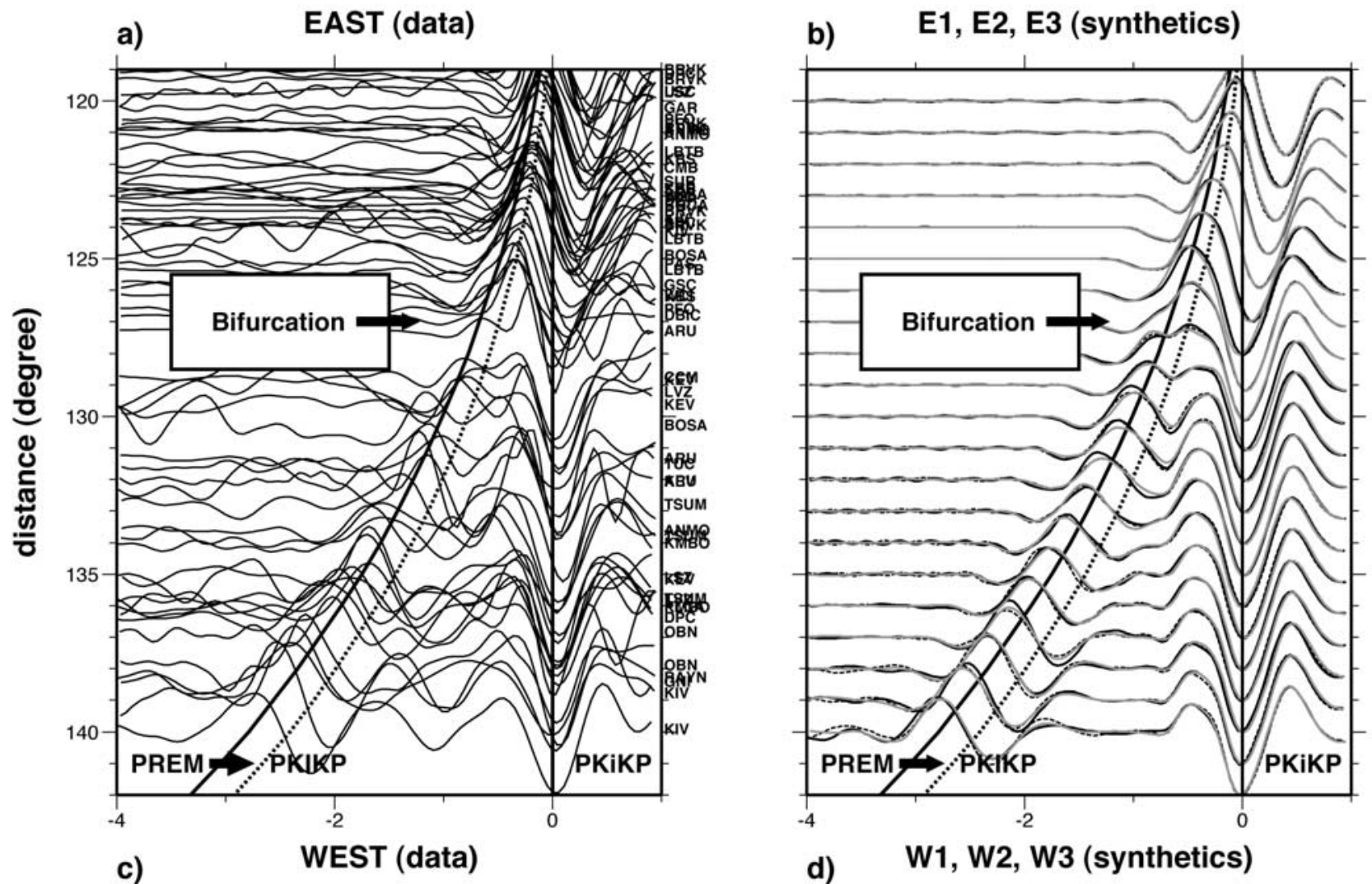

d) W1, W2, W3 (synthetics)
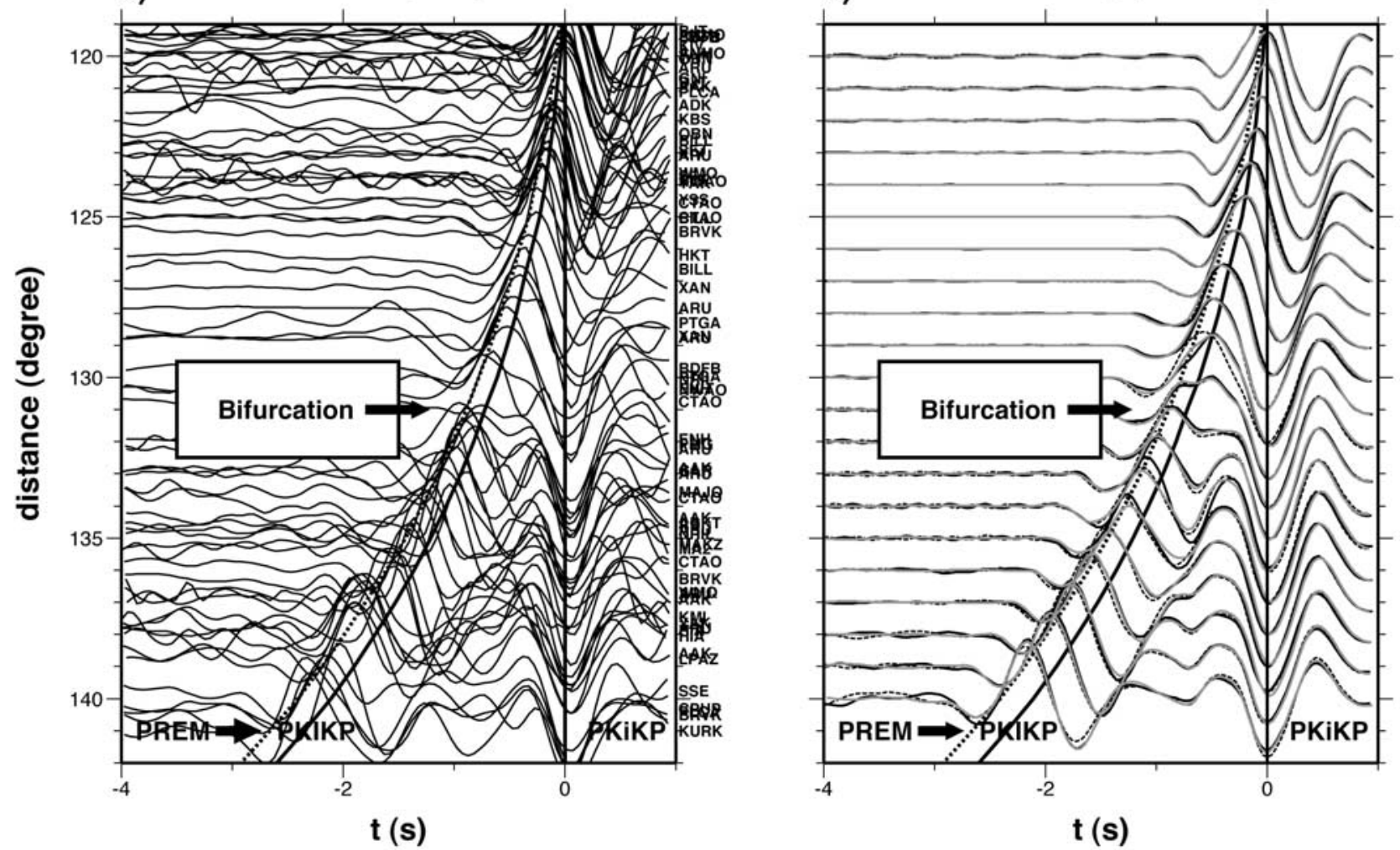

Figure 7 

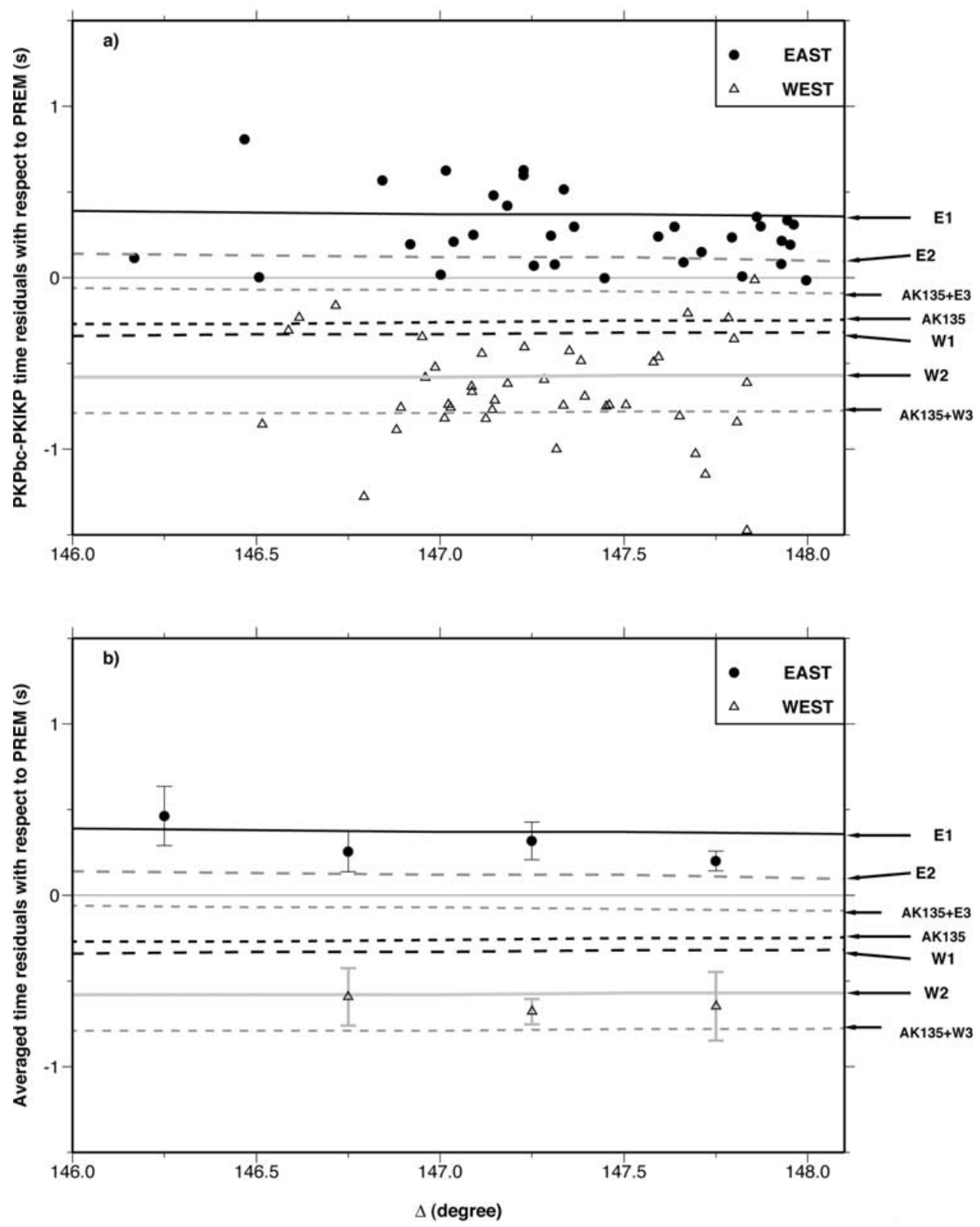

Figure 8. Same as Figure 5, except with tomographic corrections using a $P$ velocity model by van der Hilst et al. [1997].

Figure 7. Observed waveforms for the PKIKP and PKiKP phases sampling the (a) eastern and (c) western hemispheres recorded in the GSN stations from Wen and Niu [2002]. Synthetic waveforms calculated on the basis of (b) E1 (black solid traces), E2 (black dashed traces), AK135+E3 (gray solid traces) and (d) W1 (black solid traces), W2 (black dashed traces), AK135+W3 (gray solid traces). The maximum PKIKP amplitudes are impossible to pick at distances less than $127^{\circ}$ for those sampling the eastern hemisphere (Figure 7a) and at distances less than $130^{\circ}$ for those sampling the western hemisphere (Figure 7c). For those distances, synthetics are aligned along the predicted PKIKP phases on the basis of E1 (Figure 7b) and W1 (Figure 7d). The seismic data are aligned by waveform fitting the synthetics (Figure 7a is based on Figure 7b; Figure 7c is based on Figure 7d). At larger distances, synthetics and seismic data are aligned along the predicted and handpicked maximum PKiKP amplitudes, respectively. Distance corrections are applied to a common source depth of $600 \mathrm{~km}$. Accordingly, synthetics are calculated on the basis of a source depth of $600 \mathrm{~km}$. PKIKP arrivals predicted by PREM and E1, W1 are indicated by the dotted and solid lines, respectively. Note that different velocity structures at the bottom of the outer core among models PREM, OW, and AK135 can generate indistinguishable synthetics by modifying seismic velocity structures in the top of the inner core. 
a)

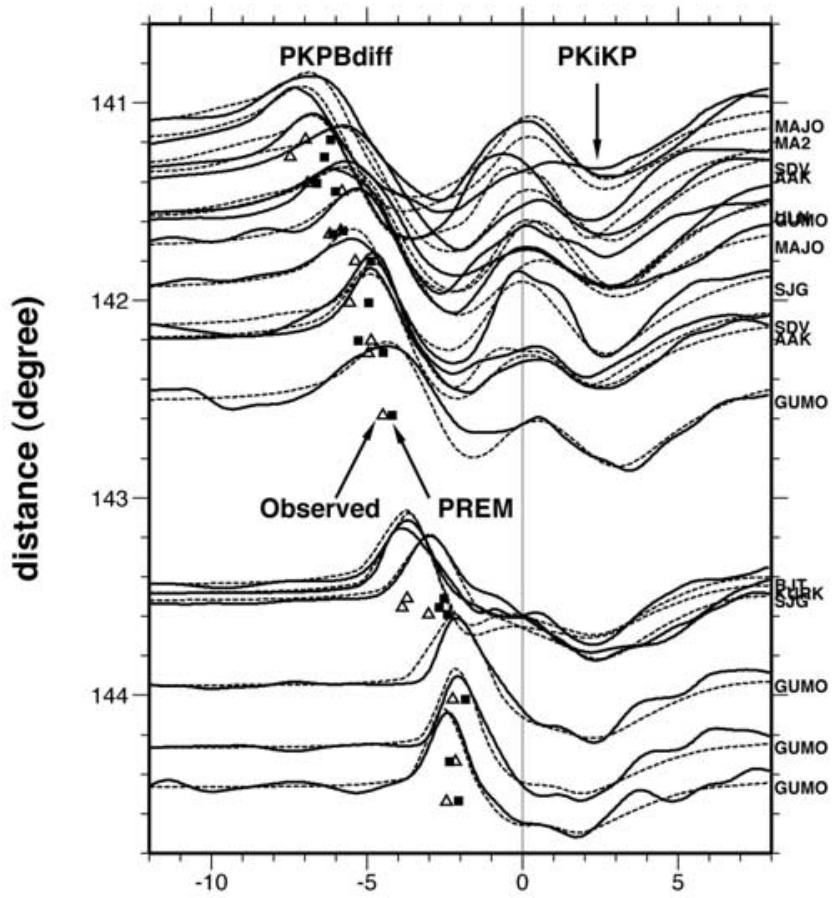

c)

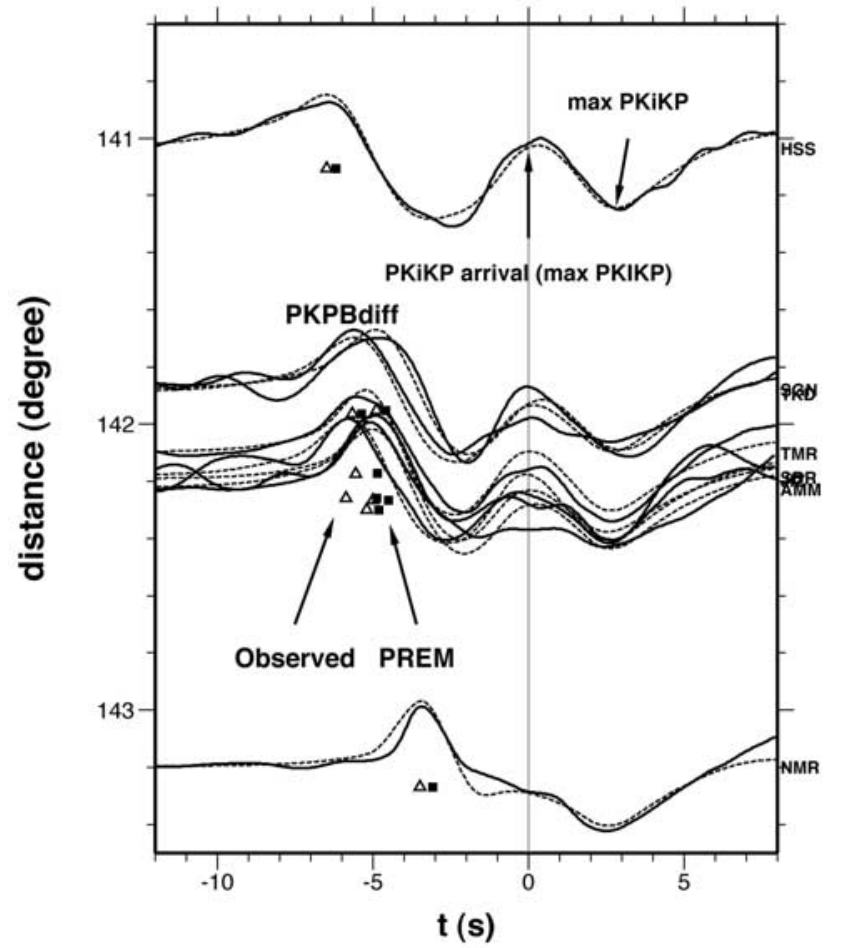

b)

EAST (global)

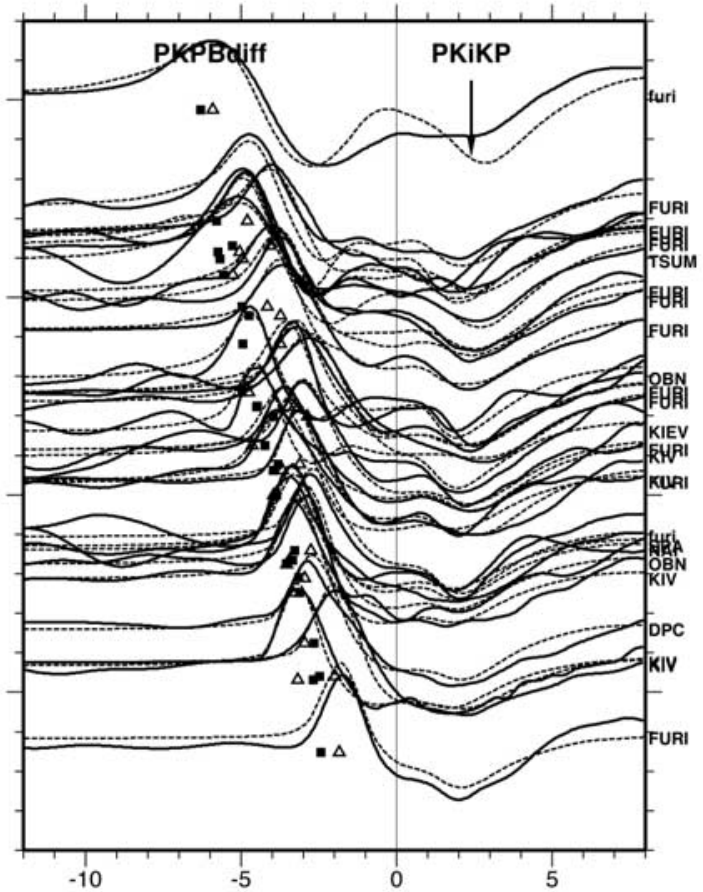

d)

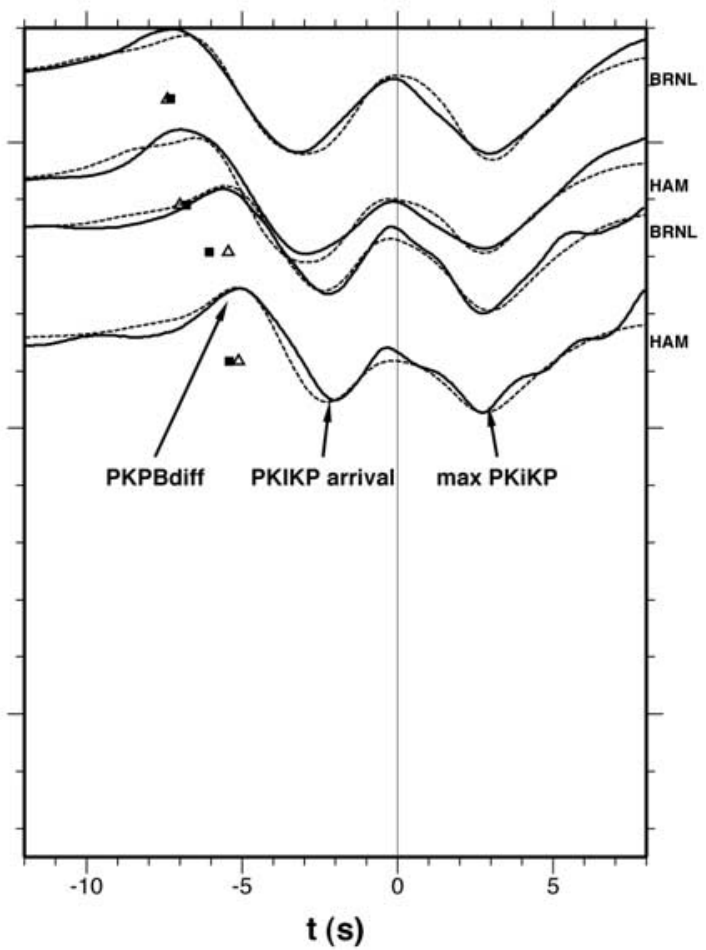

Figure 9. Observed seismograms (heavy traces) recorded by (a, b) the GSN stations and two regional arrays (c) FREESIA and (d) GRF at the $B$ caustics along with the corresponding synthetic seismograms (dashed traces) (see Figure 10 for the magnitude of the residuals and geographic locations of the bottom of the outer core sampled by the PKiKP phases). All broadband seismograms are filtered with the WWSSN long-period instrumental response and are aligned along the PKiKP arrivals predicted by PREM. Each synthetic seismogram is calculated on the basis of the source time function, epicentral distance, and source depth of its associated observation. Open triangles and solid squares represent the observed $P K P B_{\text {diff }}$ maximum amplitudes and predicted ones on the basis of PREM, respectively. All traces are distance corrected to a common source depth of $600 \mathrm{~km}$. 


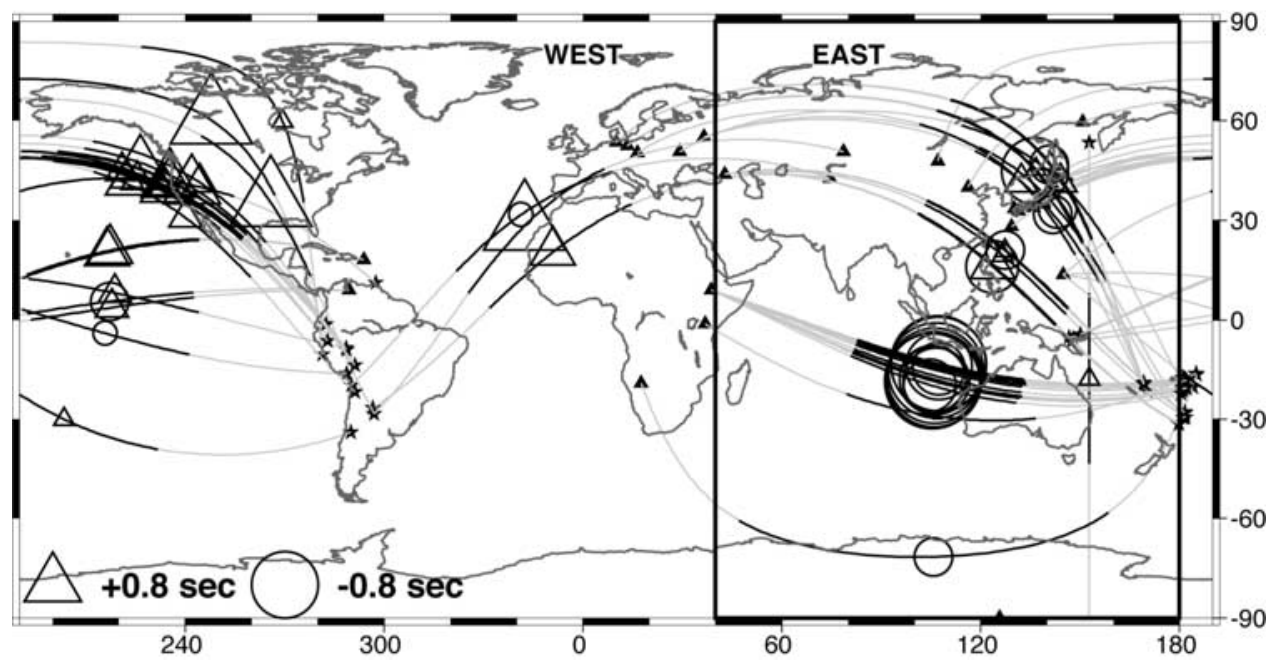

Figure 10. Map view of $P K i K P$ and $P K P B_{\text {diff }}$ great circle paths, travel time residuals displayed as symbols centered at the $P K i K P$ bouncing points, and the $P K i K P$ ray segments through the bottom $200 \mathrm{~km}$ of the outer core (heavy lines). Circles and triangles represent negative and positive residuals with respect to PREM, respectively. The magnitude of residuals is proportional to the size of the symbols. Stars and triangles indicate the locations of earthquakes and stations, respectively.

$P K P b c-P K I K P$ data sets, but the corrections cannot effectively reduce the scatter of the data (Figure 12).

\subsection{Seismic Observations of the PKPab-PKPbc Phases at $146^{\circ}-159^{\circ}$}

[13] In this section, we use the $P K P a b-P K P b c$ differential travel times and the $P K P b c / P K P a b$ amplitude ratios observed at $146^{\circ}-159^{\circ}$ to study seismic velocity structure in the outer core. We select 406 PKPab-PKPbc waveforms from about 3600 observations recorded in the GSN, BANJO, BLSP, FREESIA, GRF, and GRSN. Our geographic coverage is reasonably good (Figure 13). Differential travel times are determined by the time difference between the handpicked maximum amplitudes of the short-period $P K P b c$ phase and the Hilbert-transformed short-period PKPab phase. The observed time residuals are scattered for both hemispheres (Figure 14). We average the time residuals every $1^{\circ}$ of the epicentral distance. The differential travel time residuals and averages do not show any pattern. However, these differential travel time residuals would reject $\mathrm{LM}$, the alternative model for explaining the observed $P K i K P-P K P B_{\text {diff }}$ waveforms sampling the western hemisphere (Figure 14). Differential travel times of PKPab$P K P b c$ would be affected by the seismic structure in the

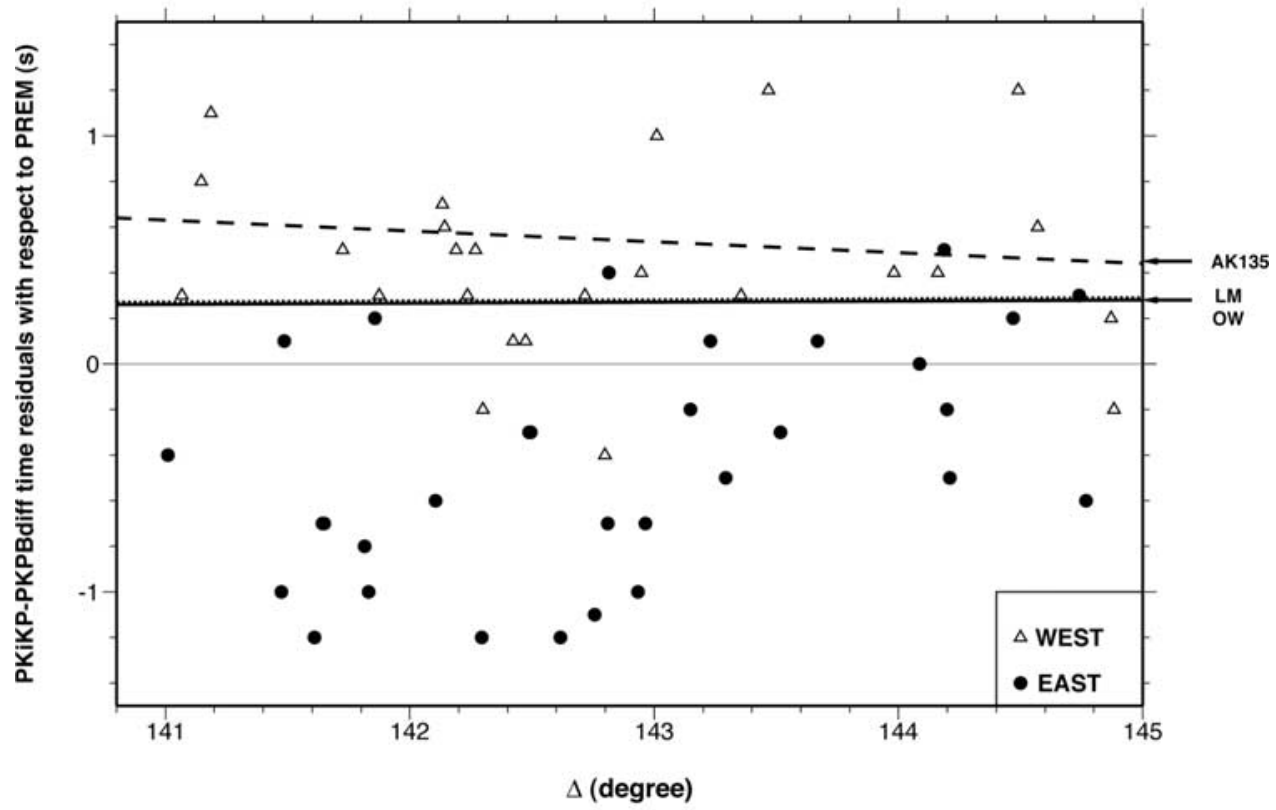

Figure 11. Observed $P K i K P-P K P B_{\text {diff }}$ differential travel time residuals with respect to PREM plotted as a function of epicentral distance, along with the predictions from AK135, OW, and LM. 


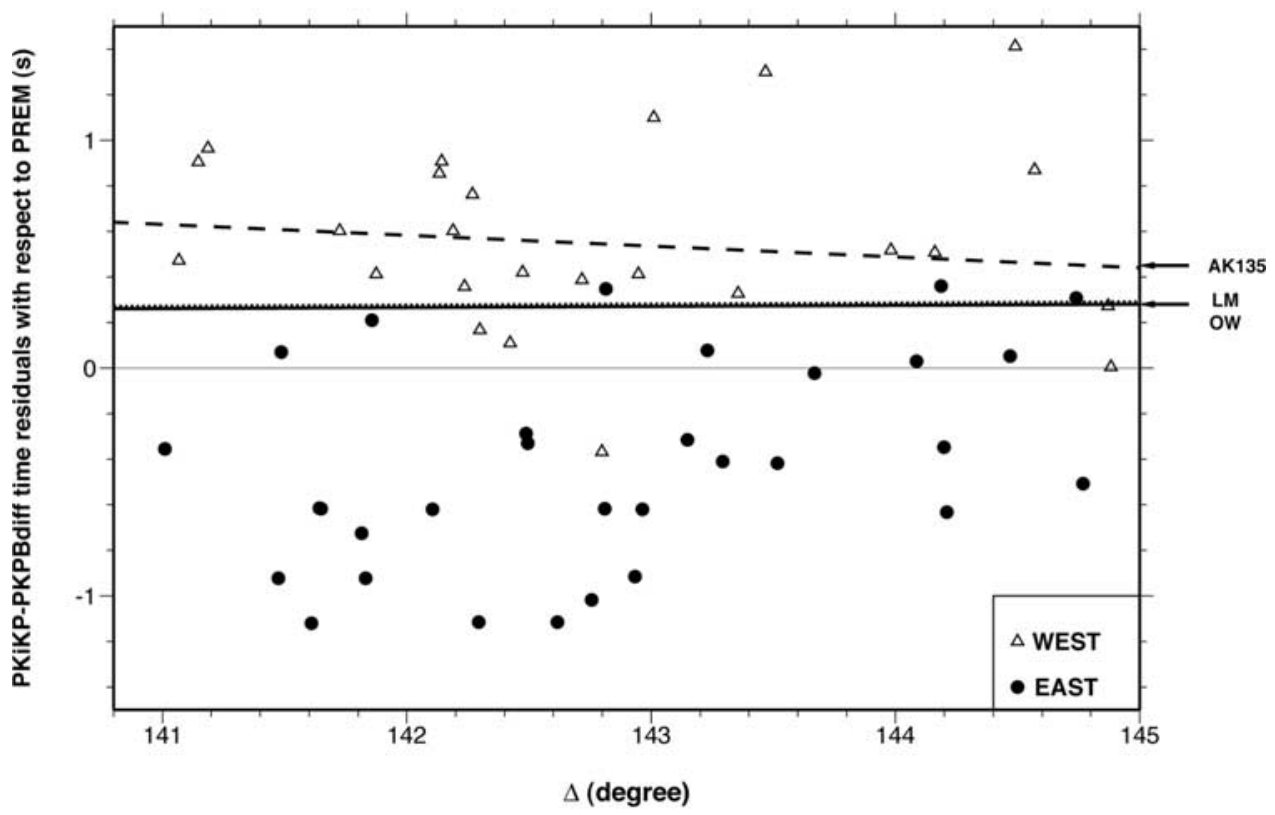

Figure 12. Same as Figure 11, except that the travel time residuals are corrected using a $P$ velocity tomographic model by van der Hilst et al. [1997].

lower mantle, the bottom of the outer core, and the top of the outer core. The contributions to the differential travel time anomalies from the top portion of the outer core are likely small because of small or no seismic velocity variations there. For example, Tanaka and Hamaguchi [1993] analyzed differential travel times of $\operatorname{SmKS}(\mathrm{m}=1,2,3)$ and proposed $\pm 0.3 \%$ variations in seismic velocity in the top $200 \mathrm{~km}$ of the liquid outer core. These small variations of seismic velocity would predict a maximum of $0.05 \mathrm{~s}$ of $P K P a b-P K P b c$ differential travel time residual. At epicentral distances larger than $149^{\circ}$, when the $P K P b c$ waves turn in the bottom $200 \mathrm{~km}$ of the outer core, the predicted differential travel time residuals from PREM and OW start to deviate by $0.4 \mathrm{~s}$ (Figure 14). This travel time difference reflects the effect of different velocity gradients between the two models at the bottom of the outer core. It appears that the averages of the travel time residuals observed at large epicentral distances would reject $\mathrm{OW}$ at the bottom of the outer core (Figure 14b). We however note that PREM underpredicts the averages of the observed travel time residuals at the distances before $149^{\circ}$. This is caused by the PREM seismic structures in the mantle and in the top portion of the outer core. If we modify PREM seismic structures in the mantle and in the top portion of the outer

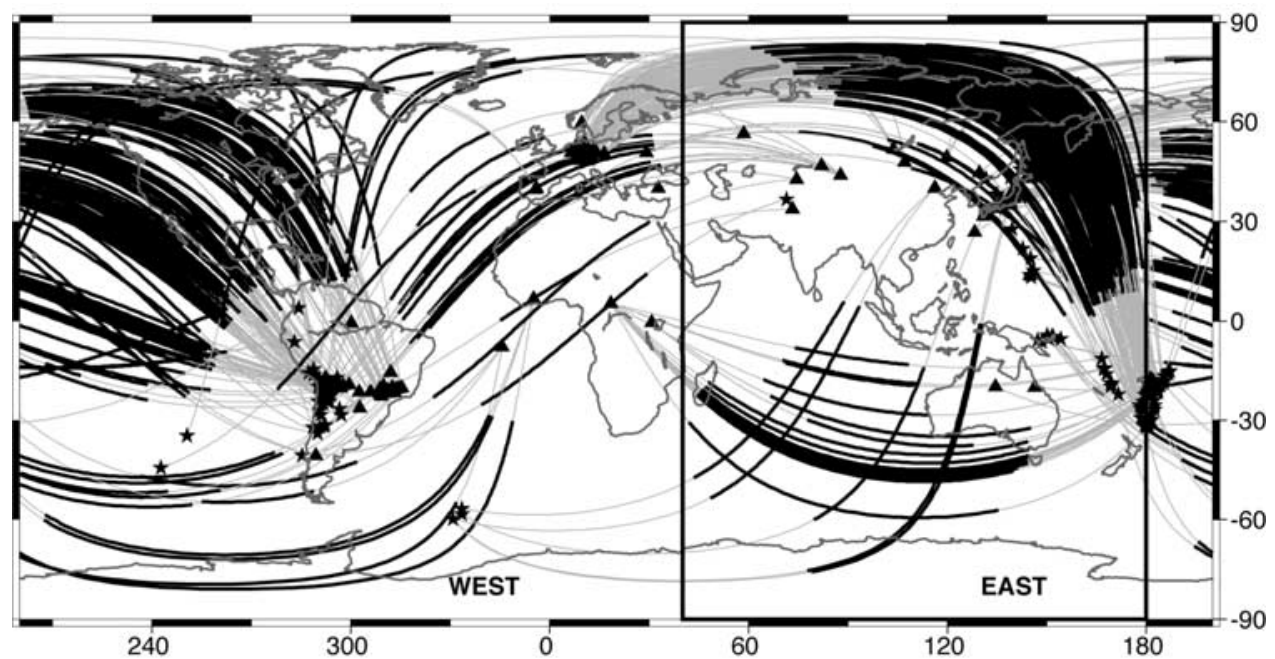

Figure 13. Map view of great circle paths (gray lines) and ray segments (black lines) of the $P K P b c$ phases sampling the bottom $400 \mathrm{~km}$ of the outer core collected from the stations in the GSN and several regional arrays in South America (BANJO, BLSP), Japan (FREESIA), and German (GRF, GRSN). Stars and triangles indicate the locations of events and stations, respectively. The geographic separation of the two "hemispheres" is also marked. 

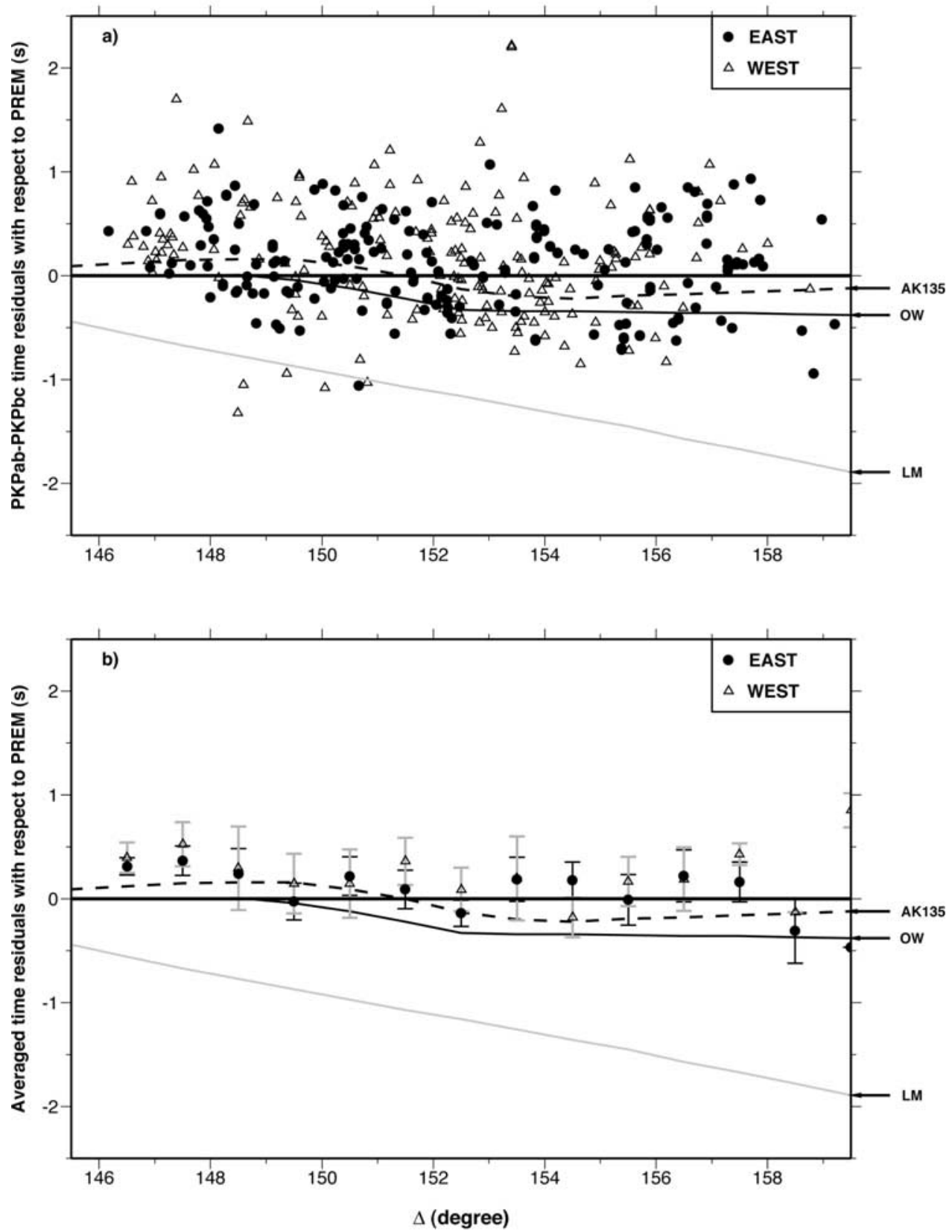

Figure 14. (a) Observed $P K P a b-P K P b c$ differential travel time residuals and (b) their averages over every $1^{\circ}$ with respect to PREM plotted as a function of epicentral distance, along with the predictions from AK135, OW, and LM. Standard deviations are also plotted in Figure 14b.

core to fit the averages of the travel time residuals in the closer distance range, the observed travel time averages in the larger distances neither confirm nor exclude OW or PREM structures in the bottom of the outer core. The observations also show a large magnitude of scatter over all distances of $146^{\circ}-159^{\circ}$, indicating that the PKPab$P K P b c$ differential travel times are significantly affected by the seismic heterogeneities in the lower mantle. We again introduce the $P$ wave tomographic model by van der Hilst et al. [1997] to correct the travel time residuals caused by the mantle structures. The magnitude of the travel time residual predicted by the mantle tomographic model is much larger than that predicted for the PKPbc-PKIKP data set, because of the large separations of the $P K P b c$ and $P K P a b$ ray paths in the mantle. The corrections however do not reduce data scatters, suggesting the existence of small-scale seismic heterogeneities in the mantle (Figure 15). The large scatters of the PKPab-PKPbc differential travel times make it impossible to use this data set to resolve the velocity structure at the bottom of the outer core. Detailed modeling of the effect of the seismic anomalies in the mantle on the $P K P a b-P K P b c$ travel times is beyond the discussion of this paper. We note that the current $P$ wave mantle tomographic models only marginally explain the scatter of the differential travel times of the PKPab-PKPbc and PKPab-PKIKP data sets [Breger et al., 2000; Ishii et al., 2002]. 

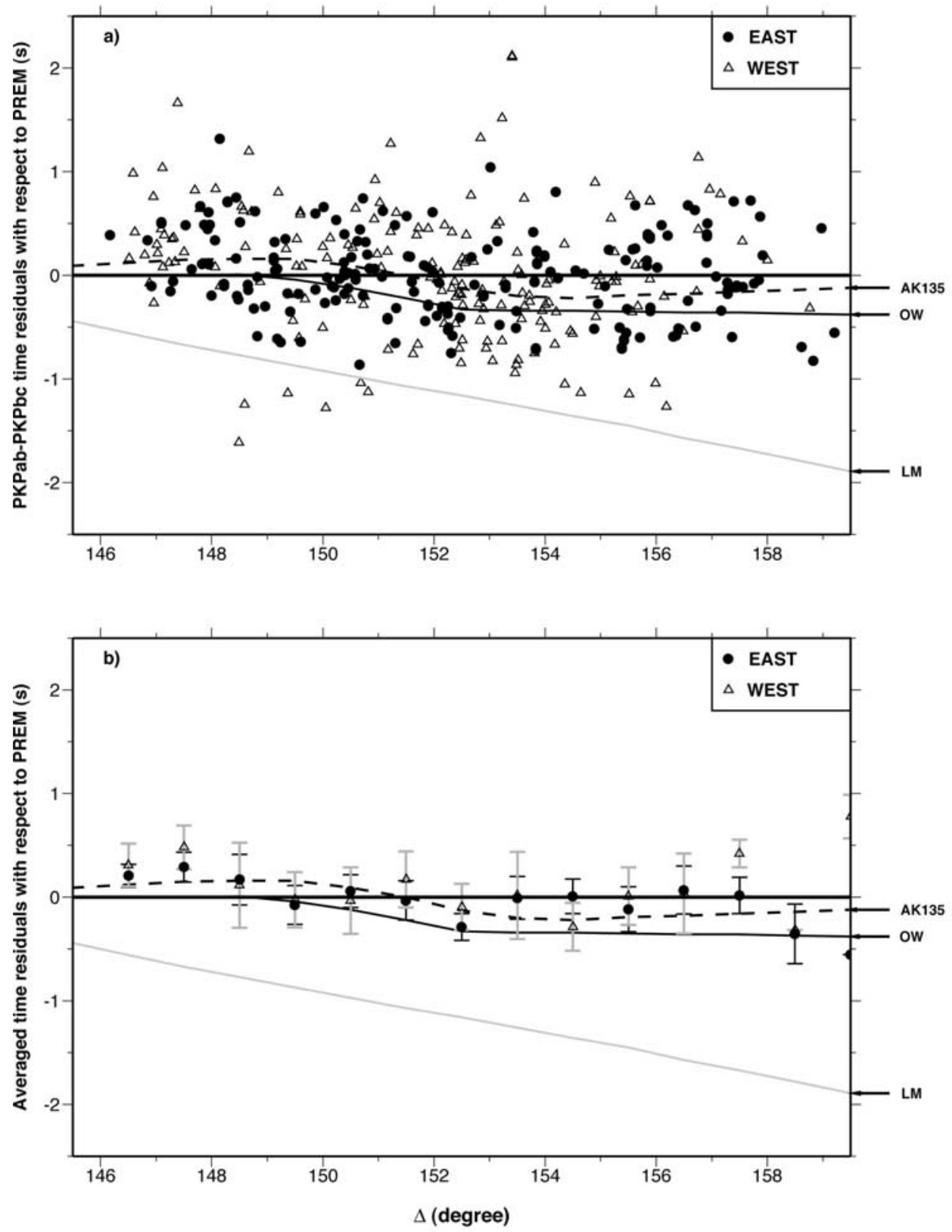

Figure 15. Same as Figure 14, except that the travel time residuals are corrected using a $P$ velocity model by van der Hilst et al. [1997].

[14] $P K P b c / P K P a b$ amplitude ratios could provide an additional constraint on the seismic velocity structure at the bottom of the outer core. When the $P K P b c$ waves diffract along the ICB $\left(\Delta>152^{\circ}\right)$, a low-velocity gradient at the bottom of the outer core would generate a stronger $P K P b c$ phase and thus a larger $P K P b c / P K P a b$ amplitude ratio than the PREM gradient. We calculate synthetic seismograms and handpick the maximum amplitudes of the $P K P b c$ phase and the Hilbert-transformed $P K P a b$ phase on the basis of PREM and OW. Note that OW predicts larger $P K P b c / P K P a b$ amplitude ratios at the epicentral distances larger than $152^{\circ}$ (Figure 16). The observed $P K P b c / P K P a b$ amplitude ratios and the averaged amplitude ratios show large scatters (Figure 16). The large scatters prevent us from distinguishing different velocity gradients at the bottom of the outer core on the basis of the amplitude ratio data. The scatters of the PKPbc/PKPab amplitude ratios reflect the uncertainties in the earthquake focal mechanism determination and, perhaps more likely, the scattering of small-scale seismic heterogeneities elsewhere.

\subsection{Seismic Velocity Models in the Outer Core}

[15] Our preferred seismic velocity models, mainly derived from the joint modeling of the PKiKP-PKIKP waveforms and the $P K P b c-P K I K P$ differential travel times and waveforms, suggest different radial velocity gradients at the bottom of the outer core in the two hemispheres. The model in the eastern hemisphere has PREM velocities (with a radial velocity gradient of $0.114(\mathrm{~km} / \mathrm{s}) / 200 \mathrm{~km})$ at the bottom of the outer core, followed by a velocity increase 

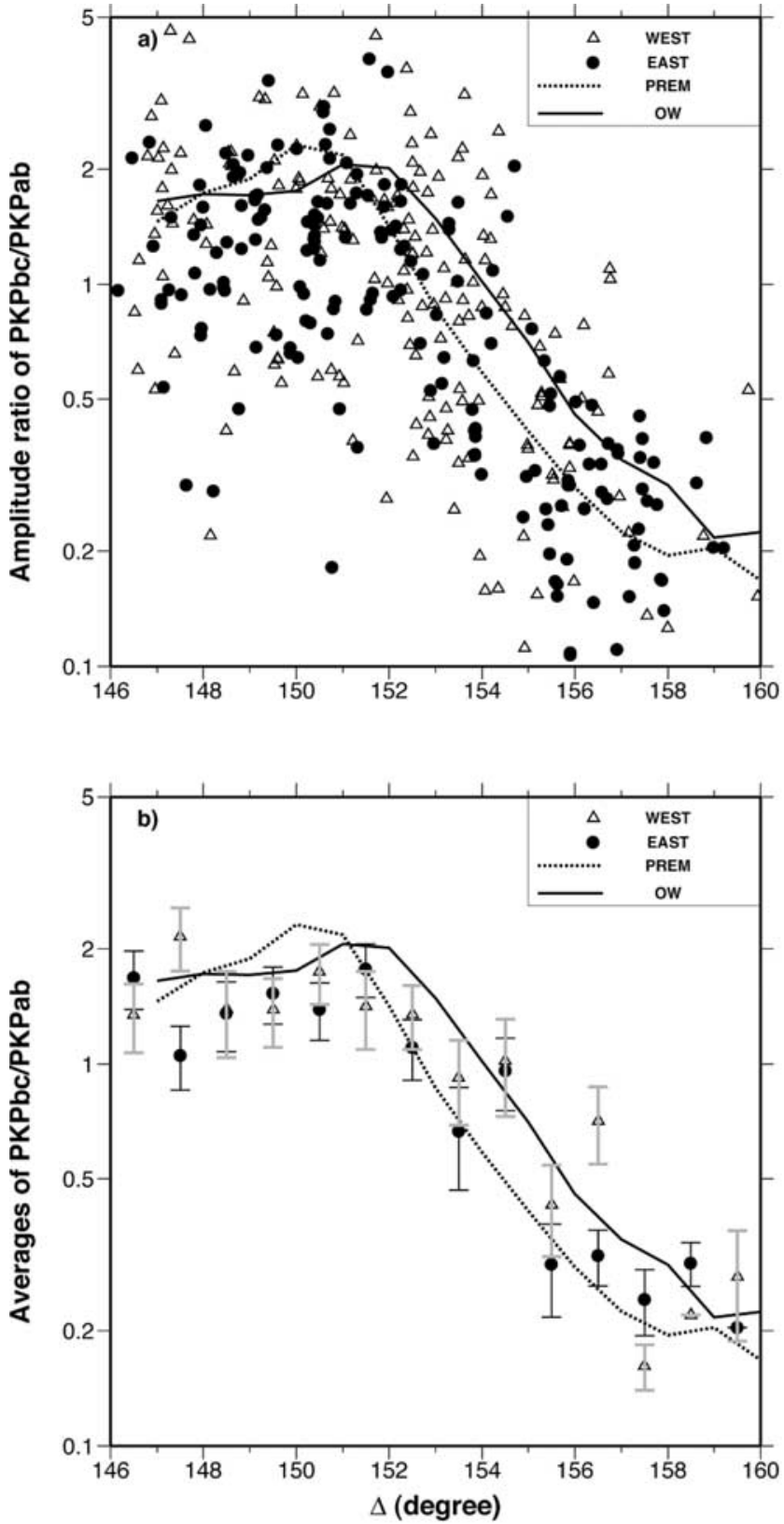

Figure 16. (a) Observed $P K P b c / P K P a b$ amplitude ratios and (b) their averages over $1^{\circ}$ in semilog scale plotted as a function of epicentral distance, along with the predictions by PREM and OW for a source depth of $100 \mathrm{~km}$. The observed amplitude ratios are corrected for the radiation patterns of the earthquake sources.

of $0.748(\mathrm{~km} / \mathrm{s})$ across the ICB. The model in the western hemisphere, OW, has a lower-velocity gradient of $0.0083(\mathrm{~km} / \mathrm{s}) / 200 \mathrm{~km}$ at the bottom $200 \mathrm{~km}$ of the outer core and a velocity increase of $0.645(\mathrm{~km} / \mathrm{s})$ across the ICB (Figures 6 and 17b). We now discuss the evolution of the outer core models and the low-velocity gradients in the bottom of the outer core appearing in AK135 and PREM2.

[16] Several early proposed solutions at the bottom of the outer core are quite different, such as those by Jeffreys [1939], Bolt [1962, 1964], Adams and Randall [1964], and model 132 [Buchbinder, 1971]. Those models were, however, mainly derived by fitting the PKIKP precursors that were later proved to be caused by the scattering near the $\mathrm{CMB}$, rather than at the bottom of the outer core [Cleary and Haddon, 1972; Haddon and Cleary, 1974]. The differences among the subsequent models were resulted from different types of seismic data sets used in deriving the models. For example, PEM [Dziewonski et al., 1975], C2 [Anderson and Hart, 1976], and PREM were derived from free oscillations and travel times; KOR5 [Qamar, 1973] was derived from travel times, amplitudes of core phases, and multiple reflected core phases; PREM2 was obtained from fitting travel times, amplitude ratios, and waveforms of various PKP branches; IASP91 [Kennett and Engdahl, 1991] and AK135 were derived from fitting travel times; and PMNA [Song and Helmberger, 1992] was derived from waveform modeling (Figure 17a).

[17] Here, we attempt to assess the flat velocity gradient at the bottom of the outer core appearing in AK135 and PREM2 by jointly modeling the PKiKP-PKIKP waveforms and the $P K P b c-P K I K P$ differential travel times and waveforms. Because AK135 and PREM2 have similar seismic structures in the bottom of the outer core, we only present the results for AK135. We fix the seismic structures in the mantle and outer core to be AK135 and change the ICB depth to be that in PREM. We then modify the velocity jumps across the ICB and velocities in the top of the inner core until their synthetics explain the observed PKiKPPKIKP waveforms. The resultant inner core models E3 (for the eastern hemisphere) and W3 (for the western hemisphere) are shown in Figure $17 \mathrm{~b}$ and their PKiKPPKIKP synthetic waveforms are shown in Figure 7. The coupled outer core-inner core models AK135+E3 and AK135+W3 predict $P K P b c-P K I K P$ travel time residuals about $0.5 \mathrm{~s}$ and $0.3 \mathrm{~s}$ smaller than the observations sampling the eastern and western hemispheres, respectively (Figure 5). These misfits cannot be reconciled by assuming different velocity gradients in the top of the inner core either. If we fix the velocity jumps across the ICB to be those of E3 and W3, which are required to explain the bifurcation distances and the waveforms observed in the bifurcation distance ranges for the PKiKP-PKIKP data sampling the eastern and western hemispheres respectively, and increase the velocity gradients in the top of the inner core to explain the $P K P b c$ $P K I K P$ differential travel times, the modified models would predict larger PKIKP and PKIKP time separations than the observations at larger distances. The low velocities of AK135 and PREM2 in the bottom of the outer core appear to be too low to consistently explain the seismic data sampling the both hemispheres.

\subsection{Other Possible Seismic Structures Within the Outer Core}

[18] In this section, we search other possible seismic velocity structures in the liquid outer core. The assembled data do not show any pattern with respect to their turning longitude, turning latitude, azimuth, and ray angle of those core phases. We present here a more detailed analysis of possible existence of seismic anomalies in the tangential cylinder.

[19] Many geodynamic models [e.g., Glatzmaier and Roberts, 1995; Kuang and Bloxham, 1997] indicate that 

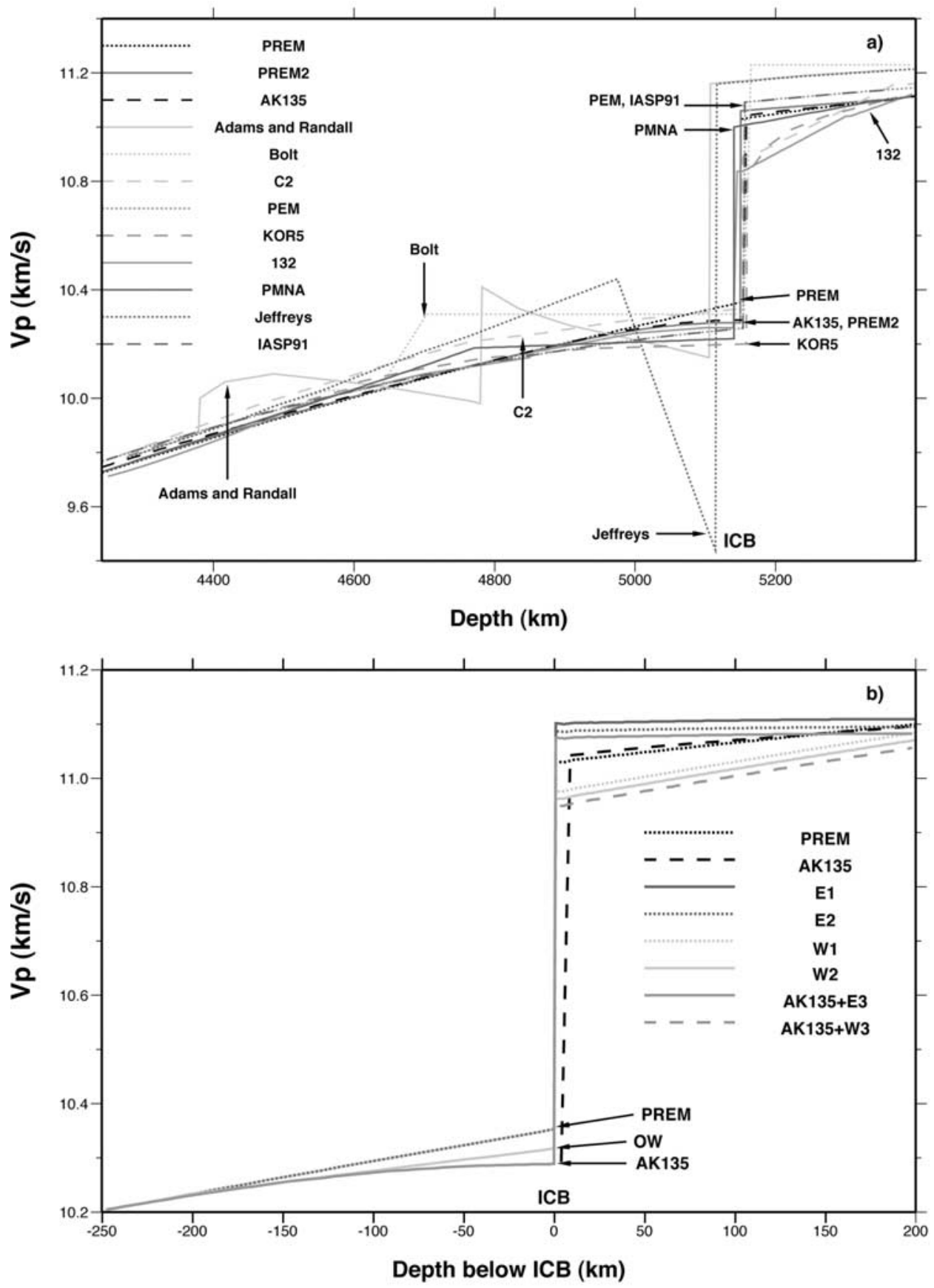

Figure 17. (a) Previous proposed $P$ wave velocity models of the Earth's core: PREM, PREM2, AK135, Adams and Randall [1964], Bolt [1962, 1964], C2 [Anderson and Hart, 1976], PEM [Dziewonski et al., 1975], KOR5 [Qamar, 1973], 132 [Buchbinder, 1971], PMNA [Song and Helmberger, 1992], Jeffreys [1939], and IASP91 [Kennett and Engdahl, 1991]. (b) PREM, AK135, and the coupled outer core-inner core models of this study: E1, W1, E2, W2, AK135+E3, and AK135+W3. See color version of this figure in the HTML.

there may exist a columnar convection in the outer core within the so-called tangential cylinder, a cylinder that circumscribes the inner core. It is generally believed that the convection pattern inside the tangential cylinder is different from the rest of the outer core. Some authors [Aurnou et al., 1996] estimate that the temperature inside the tangential cylinder is about $0.001 \mathrm{~K}$ higher than that outside the cylinder, and this extremely small-magnitude of temperature difference can drive the convection in the outer core and sustain the Earth's magnetic field. This magnitude of temperature variation would directly have a negligible effect on seismic velocity. However, the temperature variation or different types of convection may introduce compositional difference or anisotropy inside the tangential cylinder, which may have an observable effect on the seismic velocity there. We investigate possible seismic 

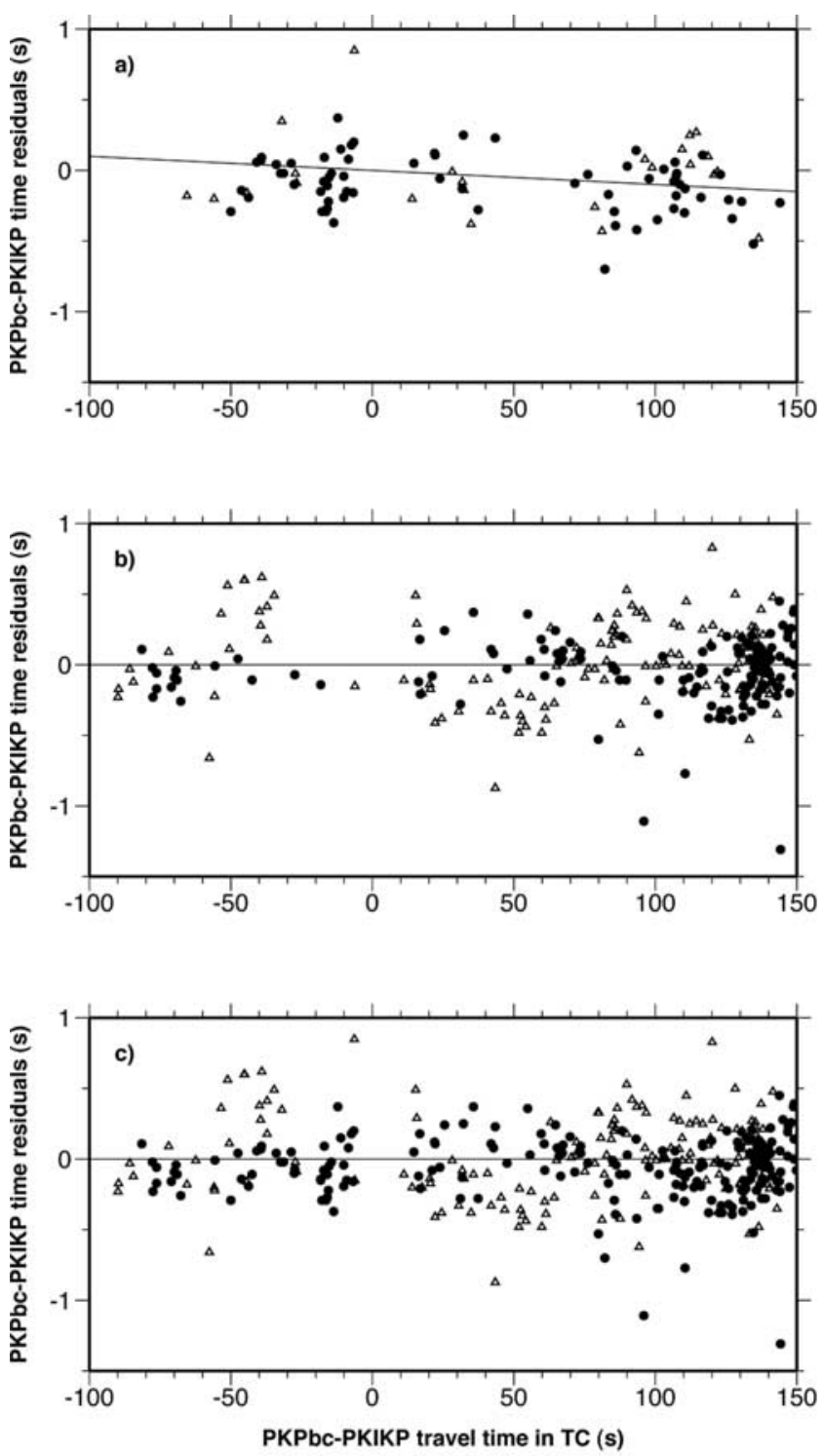

Figure 18. Differential $P K P b c-P K I K P$ travel time residuals as a function of the difference between the times of $P K P b c$ and PKIKP travel in the tangential cylinder (TC). Depending on the PKIKP sampling regions in the inner core, the travel time residuals are calculated with respect to E1 (for those sampling the eastern hemisphere) and W2 (for those sampling the western hemisphere) [Yu et al., 2003], respectively. The data are displayed according to the PKIKP ray angles with respect to the equatorial plane: (a) $0^{\circ}-30^{\circ}$, (b) $30^{\circ}-45^{\circ}$, and (c) $0^{\circ}-45^{\circ}$. Solid circles and open triangles represent the data for those PKIKP phases sampling the inner core beneath the eastern and western hemispheres, respectively. The solid lines are the predicted time residuals within the tangential cylinder being $0.1 \%$ faster than PREM (Figure 18a) and PREM-like (Figures 18b and $18 \mathrm{c})$, respectively.

velocity structure within the tangential cylinder by analyzing the differential travel times of the PKPbc-PKIKP at the distance range of $147^{\circ}-153^{\circ}$. We select the data on the basis of the following criteria: 1) their PKIKP ray angles are smaller than $45^{\circ}$ from the equatorial plane to avoid the influence from the inner core anisotropy; and 2) their PKIKP ray paths are confined in each of the hemispheres of the inner core to avoid east-west lateral variations in the inner core. At this distance range, the differential travel times are affected by the seismic structures in the top $400 \mathrm{~km}$ of the inner core. To eliminate the effect of seismic heterogeneities in the inner core and in the bottom of the outer core, the differential travel time residuals are calculated with respect to E1 and W2 from Yu et al. [2003] for the data sampling the inner core beneath the eastern and western hemispheres, respectively. Note that we are able to extend E1 and W2 seismic velocity models to the deeper portion of the inner core by jointly modeling the PKPbc-PKIKP differential travel times at the distance range of $146^{\circ}-$ $159^{\circ}$ and the PKiKP-PKIKP waveforms. Figure 18 shows the differential $P K P b c-P K I K P$ travel time residuals as a function of the difference between the times spent by the $P K P b c$ wave and the PKIKP wave in the tangential cylinder. We group the selected data into three subsets according to the $P K I K P$ ray angles with respect to the equatorial plane: $0^{\circ}-30^{\circ}$ (Figure $18 \mathrm{a}$ ), $30^{\circ}-45^{\circ}$ (Figure $18 \mathrm{~b}$ ), and $0^{\circ}-45^{\circ}$ (Figure 18c). A negative (positive) correlation between the differential $P K P b c-P K I K P$ travel time residuals with the time difference that $P K P b c$ and PKIKP travel in the tangential cylinder would suggest that the tangential cylinder is faster (slower) than the outer core outside the tangential cylinder, while no correlation would indicate that the tangential cylinder has the same velocity as the surrounding outer core. We estimate the possible velocity perturbation in the tangential cylinder by searching the best fitting model to explain the $P K P b c-P K I K P$ differential travel time residuals. Our results suggest that the standard deviation of the $P K P b c-P K I K P$ differential travel time residuals for the $P K P b c$ and $P K I K P$ phases sampling the tangential cylinder is the smallest when the tangential cylinder is $0.1 \%$ faster than PREM for the observations whose ray angles are between $0^{\circ}-30^{\circ}$ (Figure 18a), PREMlike for those whose ray angles are $30^{\circ}-45^{\circ}$ (Figure 18b) and $0^{\circ}-45^{\circ}$ (Figure 18c). We conclude that the velocity perturbation in the tangential cylinder, if it exists, is less than $0.1 \%$.

\section{Possible Implications}

[20] Different velocity gradients in the bottom of the outer core may indicate the existence of large-scale temperature difference and/or compositional change there. From a compositional point of view, it is natural to relate the different velocity gradients to density difference at the bottom of the outer core, and ultimately to different enrichments of light elements such as, $\mathrm{S}, \mathrm{O}, \mathrm{H}, \mathrm{Si}$, etc. Laboratory experiments [Anderson and Ahrens, 1994] suggested that the isentropic bulk modulus, under core conditions, is independent of temperature and pressure. If the bulk modulus is indeed constant, $P$ velocity would be inversely proportional to the square root of density. The velocity structure of $\mathrm{OW}$, which has reduced velocities relative to PREM linearly increasing from $0 \%$ at $200 \mathrm{~km}$ above the ICB to $-0.35 \%$ at the ICB, would suggest that the density increases relative to PREM from $0 \%$ at $200 \mathrm{~km}$ above the ICB to about $0.7 \%$ at the ICB. In other words, the bottom of the outer core beneath the 
western hemisphere is less enriched in light elements than the eastern hemisphere.

[21] From a thermodynamic point of view, a compositional difference at the bottom of the outer core between the two hemispheres could be coupled with a large-scale temperature difference there and may also be related to the hemispheric variation of seismic structures in the top of the inner core. A large-scale temperature variation may affect the freezing process, freezing rate near the ICB, and thermodynamic state in the bottom of the outer core, and thus produces a large-scale variation of enrichments in light elements in the bottom of the outer core. Different enrichments in light elements in the bottom of the outer core may be coupled with different compositions and melt behaviors in the top of the inner core, and thus provide an explanation to the hemispheric variation of seismic structures in the top of the inner core. A recent study [Wen and Niu, 2002] suggested that different geometric inclusions of melt may provide an explanation to the hemispheric variations of isotropic velocity and attenuation, and a correlation of high velocity with high attenuation in the top of the inner core beneath the eastern hemisphere.

[22] One possible explanation for a hemispheric pattern of temperature difference at the bottom of the outer core is that the thermal structure at the bottom of the outer core is induced by the heterogeneous thermal sources at the CMB, as suggested by Niu and Wen [2001] and Wen and Niu [2002] and by the laboratory experiment of Sumita and Olson [1999]. In that scenario, the life time of this largescale compositional difference at the bottom of the outer core would likely depend on that of the thermal structure at the CMB.

\section{Conclusion}

[23] We assemble various $P K P$ observations at the epicentral distance range of $120^{\circ}-159^{\circ}$ recorded at the Global Seismographic Network and several regional seismic networks to study seismic velocity structure in the Earth's outer core. The observations show these characteristics: (1) both the observed PKiKP-PKIKP and PKPbc-PKIKP differential travel times show an "east-west" hemispheric difference. PKIKP phases arrive about $0.7 \mathrm{~s}$ earlier for those sampling the "eastern" $\left(40^{\circ} \mathrm{E}-180^{\circ} \mathrm{E}\right)$ hemisphere than those sampling the "western" $\left(180^{\circ} \mathrm{W}-40^{\circ} \mathrm{E}\right)$ hemisphere along the equatorial paths; $(2)$ the observed $P K i K P-P K P B_{\text {diff }}$ differential travel times also show a hemispheric difference. PKiKP phases arrive about $0.9 \mathrm{~s}$ earlier for those sampling the "eastern" hemisphere than those sampling the "western" hemisphere; and (3) both the observed PKPab-PKPbc differential travel times and the $P K P b c / P K P a b$ amplitude ratios show scatter. The above observations can be best explained by two one-dimensional $P$ velocity models, one for each hemisphere, at the bottom of the outer core. The seismic data sampling the eastern hemisphere can be explained by PREM, while those sampling the western hemisphere can be explained by OW, which has reduced velocities relative to PREM linearly increasing from $0 \%$ at $200 \mathrm{~km}$ above the ICB to $-0.35 \%$ at the ICB. Different velocity gradients indicate that there may exist a compositional change and/or a large-scale temperature difference at the bottom of the outer core and that inner core formation processes may be different between the two hemispheres. Different inner core formation processes between the two hemispheres may produce different enrichments of light elements at the bottom of the outer core and different geometric inclusions of melt at the top of the inner core, which may provide an explanation to the seismic structures in the bottom of the outer core and in the top of the inner core. Low velocities at the base of the outer core in AK135 appear to be too low to be able to consistently explain the observed PKiKP-PKIKP waveforms and the PKPbc-PKIKP travel times for the both hemispheres. The seismic data also suggest that the variation of seismic velocity in the tangential cylinder of the outer core, if it exists, is less than $0.1 \%$. We use a $P$ velocity tomographic model to correct the travel time delays caused by the seismic heterogeneities in the mantle. The tomographic model is unable to reduce the scatter of the travel time data, suggesting the presence of unknown small-scale seismic anomalies in the mantle.

[24] Acknowledgments. We are grateful to IRIS, FREESIA, BANJO, BLSP, GRF, and GRSN for providing the waveform data. We thank Robert van der Hilst for making his $P$ wave tomographic model available. Discussions and comments from Dan Davis, Bill Holt, Don Weidner, Jennifer Kung, Shu-Huei Hung, Bor-Shouh Huang, and Ban-Yuan Kuo were helpful in preparing the manuscript. Careful proofreading by Elliot Klein improved the readability of the manuscript. Critical reviews by Ken Creager, an anonymous reviewer, and an Associate Editor improved the presentation of this paper. This work is supported by NSF grant EAR0207746.

\section{References}

Adams, R. D., and M. J. Randall (1964), The fine structure of the Earth's core, Bull. Seismol. Soc. Am., 54, 1299-1313.

Anderson, D. L., and R. S. Hart (1976), An Earth's model based on free oscillations and body waves, J. Geophys. Res., 81, 1461-1475.

Anderson, W. W., and T. J. Ahrens (1994), An equation of state for liquid iron and implications for the Earth's core, J. Geophys. Res., 99, 42734284 .

Aurnou, J. M., D. Brito, and P. L. Olson (1996), Mechanics of inner core super-rotation, Geophys. Res. Lett., 23, 3401-3404.

Bolt, B. A. (1962), Gutenberg's early PKP observations, Nature, 196, 122 124

Bolt, B. A. (1964), The velocity of seismic waves near the Earth's center, Bull. Seismol. Soc. Am., 54, 191-208.

Breger, L., H. Tkalcic, and B. Romanowicz (2000), The effects of $D^{\prime \prime}$ on $P K P(A B-D F)$ travel time residuals and possible implications for inner core structure, Earth Planet. Sci. Lett., 175, 133-143.

Buchbinder, G. G. R. (1971), A velocity structure of the Earth's core, Bull. Seismol. Soc. Am., 61, 429-456.

Cleary, J. R., and R. A. W. Haddon (1972), Seismic wave scattering near the core-mantle boundary: a new interpretation of precursors to PKP, Nature, 240, 549-551.

Creager, K. C. (1999), Large-scale variations in inner core anisotropy, J. Geophys. Res., 104, 23,127-23,139.

Dziewonski, A., and D. L. Anderson (1981), Preliminary reference Earth model, Phys. Earth Planet. Inter., 25, 297-356.

Dziewonski, A. M., A. L. Hales, and E. R. Lapwood (1975), Parametrically simple Earth models consistent with geophysical data, Phys. Earth Planet. Inter., 10, $12-48$.

Garcia, R., and A. Souriau (2000), Inner core anisotropy and heterogeneity level, Geophys. Res. Lett., 27, 3121-3124.

Garnero, E. J., and D. V. Helmberger (1995), On seismic resolution of lateral heterogeneity in the Earth's outermost core, Phys. Earth Planet. Inter, $88,117-130$.

Glatzmaier, G. A., and P. H. Roberts (1995), A three-dimensional convective dynamo solution with rotating and finitely conducting inner core and mantle, Phys. Earth Planet. Inter., 91, 63-75.

Haddon, R. A., and J. R. Cleary (1974), Evidence for scattering of seismic PKP waves near the core-mantle boundary, Phys. Earth Planet. Inter., 8, $211-234$.

Helmberger, D. V. (1983), Theory and application of synthetic seismograms, in Earthquakes: Observation, Theory and Interpretation, edited by H. Kanamori, pp. $173-222$, Soc. It. di Fis., Bologna, Italy. 
Ishii, M., A. M. Dziewonski, J. Tromp, and G. Ekström (2002), Joint inversion of normal mode and body wave data for inner core anisotropy: 2. Possible complexities, J. Geophys. Res., 107(B12), 2380, doi:10.1029/ 2001JB000713

Jeffreys, H. (1939), The times of the core waves (second paper), Mon. Not. R. Astron. Soc., Geophys. Suppl., 4, 594-615.

Kaneshima, S. (1996), Mapping heterogeneity of the uppermost inner core using two pairs of core phases, Geophys. Res. Lett., 23, 3075-3078.

Kennett, B., and E. Engdahl (1991), Traveltimes for global earthquake location and phase identification, Geophys. J. Int., 105, 429-465.

Kennett, B., E. Engdahl, and R. Buland (1995), Constraints on seismic velocities in the Earth from traveltimes, Geophys. J. Int., 122, 108-124.

Kuang, W., and J. Bloxham (1997), An Earth-like numerical dynamo model, Nature, 389, 371-374.

Luo, S., S. Ni, and D. V. Helmberger (2002), Relationship of D" structure with the velocity variations near the inner-core boundary, Geophys. Res. Lett., 29(11), 1527, doi:10.1029/2001GL013907.

Niu, F., and L. Wen (2001), Hemispherical variations in seismic velocity at the top of the Earth's inner-core, Nature, 410, 1081-1084.

Niu, F., and L. Wen (2002), Seismic anisotropy in the top $400 \mathrm{~km}$ of the inner core beneath the "eastern" hemisphere, Geophys. Res. Lett., 29(12), 1611, doi:10.1029/2001GL014118.

Ouzounis, A., and K. C. Creager (2001), Isotropy overlying anisotropy at the top of the inner core, Geophys. Res. Lett., 28, 4331-4334.

Qamar, A. (1973), Revised velocities in the Earth's core, Bull. Seismol. Soc. Am., 63, 1073-1105.

Romanowicz, B., and L. Breger (2000), Anomalous splitting of free oscillations: A reevaluation of possible interpretations, J. Geophys. Res., 105, $21,559-21,578$

Song, X., and D. V. Helmberger (1992), Velocity structure near the inner core boundary from waveform modeling, J. Geophys. Res., 97, 65736586

Song, X., and D. V. Helmberger (1995), A $P$ wave velocity model of Earth's core, J. Geophys. Res., 100, 9817-9830.

Souriau, A., and G. Poupinet (1990), A latitudinal pattern in the structure of the outermost liquid core, revealed by the travel times of $S K K S$-SKS seismic phases, Geophys. Res. Lett., 17, 2005-2007.
Souriau, A., and G. Poupinet (1991a), The velocity profile at the base of the liquid core from $P K P\left(B C+C_{\text {diff }}\right)$ data: An argument in favor of radial inhomogeneity, Geophys. Res. Lett., 18, 2023-2036.

Souriau, A., and G. Poupinet (1991b), A study of the outermost liquid core using differential travel times of $S K S, S K K S, S 3 K S$ phases, Phys. Earth Planet. Inter., 68, 183-199.

Stevenson, D. J. (1987), Limits on lateral density and velocity variations in the Earth's outer core, Geophys. J. R. Astron. Soc., 89, 311-319.

Sumita, I., and P. Olson (1999), A laboratory model for convection in Earth's core driven by a thermally heterogeneous mantle, Science, 286, $1547-1549$.

Tanaka, S., and H. Hamaguchi (1993), Degree one heterogeneity at the top of the Earth's core, revealed by $S m K S$ travel times, in Dynamics of Earth's Deep Interior and Earth Rotation, Geophys. Monogr. Ser., vol. 72, edited by J.-L. L. Mouel, D. E. Smylie, and T. Herring, pp. 127-134, AGU, Washington, D. C

Tanaka, S., and H. Hamaguchi (1997), Degree one heterogeneity and hemispherical variation of anisotropy in the inner core from $P K P(B C)-P K P$ $(D F)$ times, J. Geophys. Res., 102, 2925-2938.

van der Hilst, R. D., S. Widiyantoro, and E. R. Engdahl (1997), Evidence for deep mantle circulation from global tomography, Nature, 386, 578584

Wen, L., and F. Niu (2002), Seismic velocity and attenuation structures in the top of the Earth's inner core, J. Geophys. Res., 107(B11), 2273, doi:10.1029/2001JB000170.

Yu, W., L. Wen, and F. Niu (2003), Anomalous top layer in the inner core beneath the eastern hemisphere, Eos. Trans. $A G U, 84(46)$, Fall Meet. Suppl., Abstract S21E-0379.

F. Niu, Department of Earth Science, MS-126, Rice University, 6100 Main Street, Houston, TX 77005, USA. (niu@rice.edu)

L. Wen and W.-C. Yu, Department of Geosciences, State University of New York at Stony Brook, Stony Brook, NY 11794-2100, USA. (lianxing.wen@sunysb.edu; yu@mantle.geo.sunysb.edu) 